\title{
A global existence and uniqueness result for a stochastic Allen-Cahn equation with constraint
}

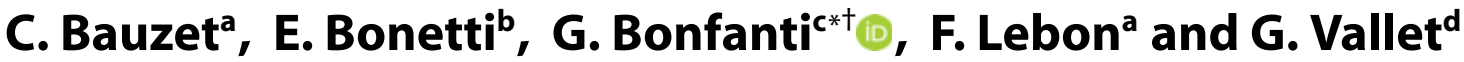

This paper addresses the analysis of a time noise-driven Allen-Cahn equation modelling the evolution of damage in continuum media in the presence of stochastic dynamics. The nonlinear character of the equation is mainly due to a multivoque maximal monotone operator representing a constraint on the damage variable, which is forced to take physically admissible values. By a Yosida approximation and a time-discretization procedure, we prove a result of global-intime existence and uniqueness of the solution to the stochastic problem.

Keywords: nonlinear parabolic equations; stochastic PDEs; existence and uniqueness

\section{Introduction}

We are interested in the following stochastic problem:

$$
\left\{\begin{aligned}
d u+(\xi-\Delta u) d t & =\left(w_{s}(u)+f\right) d t+h(u) d W & & \text { in } \Omega \times D \times(0, T) \\
u(\omega, x, t=0) & =u_{0}(x) & & \omega \in \Omega, x \in D, \\
\nabla u . \mathbf{n} & =0 & & \text { in } \Omega \times \partial D \times(0, T),
\end{aligned}\right.
$$

where $\xi \in \partial l_{[0,1]}(u), T>0, W=\left\{W_{t}, \mathcal{F}_{t}, 0 \leqslant t \leqslant T\right\}$ is a standard adapted continuous Brownian motion defined on the classical Wiener space $(\Omega, \mathcal{F}, \mathbb{P}), D$ is a smooth bounded domain of $\mathbb{R}^{d}$ with $d \geqslant 1, \mathbf{n}$ is the outward unit normal vector to $\partial D$, and $u_{0}$ is a given initial condition. Note that Equation (1.1a) can also be written in the following way:

$$
w_{s}(u)+f-\partial_{t}\left(u-\int_{0}^{t} h(u) d W\right)+\Delta u \in \partial l_{[0,1]}(u) \text { in } \Omega \times D \times(0, T),
$$

where the stochastic integral is understood in the sense of Itô.

Remark 1.1

The sub-differential $\partial l_{[0,1]}$ represents a physical constraint on $u$, which is forced to take values in the interval $[0,1]$. More precisely, we have $I_{[0,1]}: \mathbb{R} \rightarrow \mathbb{R} \cup\{+\infty\}$ defined by the following:

$$
I_{[0,1]}(x)=\left\{\begin{array}{cl}
0 & \text { if } x \in[0,1] \\
+\infty & \text { else. }
\end{array}\right.
$$

For any $x \in[0,1]$, it results that (see, e.g., [1])

$$
\partial I_{[0,1]}(x)= \begin{cases}\{0\} & \text { if } x \in] 0,1[ \\ \mathbb{R}^{-} \text {if } x=0, \\ \mathbb{R}^{+} \text {if } x=1 .\end{cases}
$$

\footnotetext{
a Aix-Marseille University, CNRS, Centrale Marseille, LMA, Marseille, France

${ }^{b}$ Department of Mathematics, University of Milan, Milan, Italy

c Department DICATAM - Section of Mathematics, University of Brescia, Brescia, Italy

d Université de Pau et des Pays de I'Adour, LMAP, UMR CNRS 5142, IPRA, Pau, France

* Correspondence to: G. Bonfanti, Department DICATAM - Section of Mathematics, University of Brescia, Brescia, Italy.

† E-mail:giovanna.bonfanti@unibs.it
} 
We assume the following hypotheses:

$H_{1}: u_{0} \in H^{1}(D)$.

$H_{2}: 0 \leqslant u_{0}(x) \leqslant 1$ for almost all $x \in D$.

$H_{3}: h: \mathbb{R} \rightarrow \mathbb{R}$ is a Lipschitz-continuous function such that $h(0)=h(1)=0$.

$H_{4}: w_{s}: \mathbb{R} \rightarrow[0,+\infty)$ is a Lipschitz-continuous function with, for convenience, $w_{s}(0)=0$.

$H_{5}: f \in \mathcal{N}_{w}^{2}\left(0, T, L^{2}(D)\right)^{\ddagger}$.

\subsection{General notations}

For the sake of clarity, let us make precise some useful notations:

- $Q=D \times(0, T)$.

- $C_{h}$ and $L_{s}$ stand for the Lipschitz constants of $h$ and $w_{s}$, respectively.

- $x . y$ the usual scalar product of $x$ and $y$ in $\mathbb{R}^{d}$.

- $V=H^{1}(D)$ and identify $H=L^{2}(D)$ with its dual space $H^{\prime}$, so that $V \hookrightarrow H \hookrightarrow V^{\prime}$ with dense and compact injections. Note that $\left(V, H, V^{\prime}\right)$ is a so-called Hilbert triplet.

- $\mathscr{D}(D)=\mathscr{C}_{c}^{\infty}(D)$ and $\mathscr{D}^{\prime}(D)$ the space of distributions on $D$.

- $\|$.$\| the usual norm in L^{2}(D)$.

- $\mathbb{E}[$.$] the expectation, that is, the integral over \Omega$ with respect to the probability measure $\mathbb{P}$.

\subsection{The model}

Equation (1.1a) is known as an Allen-Cahn-type equation, and it is used to describe several physical phenomena, like phase transitions. Here, we deal with the analysis of this equation, having in mind the evolution of damage in continuum media. More precisely, we assume that $u$ represents a damage parameter, that is, the local proportion of active cohesive bonds in the micro-structure of the material. In this direction, the function $f$ on the right hand side of (1.1a) stands for an external source of damage (mechanical or chemical). With this interpretation, we include in the model a constraint forcing $u$ to take values in the interval $[0,1]$, so that $u=1$ means that the material is completely undamaged, $u=0$ that it is completely damaged, while $u \in(0,1)$ describes an intermediate situation. The physical constraint is ensured by the presence of a sub-differential graph, that is, a multivoque maximal monotone operator. In addition, we include in the equation a multiplicative time noise where the noise diffusion coefficient $h$ depends on the damage parameter itself. From a physical point of view, the presence of a random process reflects the fact that the phenomenon of damage is related to microscopic changes in the structure and configuration of the material lattice as a consequence of breaking bonds and the formation of cavities and voids. These phenomena are clearly related to stochastic processes occurring at a microscopic level (as it is introduced in Ising materials), which we aim to take into account in a macroscopic description.

The literature on the deterministic Allen-Cahn equation is very rich, also including the presence of non-smooth (monotone) operators (see, among the others, [3-7]). For the stochastic case, we refer to fairly recent results [8,9], and the references therein. We underline that in these contributions, the sub-differential operator was replaced by smooth nonlinearities (possibly with a prescribed growth condition), as double-well potentials. However, we invite the reader interested in stochastic partial differential inclusions in a rather general situation to consult [10-12] and [13] concerning transition semigroup and invariant measures.

As an equation describing a phase transition process (as damage), (1.1a) may be recovered as a balance law referring to the theory introduced by Frémond [14]. Such a theory relies on a generalized version of the principle of virtual powers where micro-forces and micro-motions responsible for the phase transition are included. Concerning this approach, we quote the papers [15-19] where volume and surface damage models are deduced and analytically investigated. According to Frémond's theory, we now detail the derivation of system (1.1).

To this aim, we introduce as state variables of the model the damage parameter $u$ and its gradient $\nabla u$. Thus, the free energy functional reads

$$
\Psi(u, \nabla u)=\hat{w}_{s}(u)+\frac{1}{2}|\nabla u|^{2}+I_{[0,1]}(u),
$$

where the indicator function of the interval $[0,1]$ restricts the domain of the free energy to the (physically) admissible values for $u$. Moreover, the function $\hat{w}_{s}$ is related to the internal cohesion of the material, and it may depend on the damage parameter itself: Denoting by $w_{s}:=-\hat{w}_{s}^{\prime}$, we may suppose that $w_{s}$ is a positive function, vanishing once $u=0$ (the cohesion is null in the case of complete damage). Then, we introduce the dissipation of the system by a pseudo-potential of dissipation à la Moreau. As a dissipative variable, we consider the time derivative of $z=u-\int_{0}^{t} h(u) d W$. Thus, the resulting dissipation functional is as follows:

$$
\Phi\left(z_{t}\right)=\frac{1}{2}\left|z_{t}\right|^{2}
$$

${ }^{\ddagger}$ For a given separable Hilbert space $X$, we denote by $\mathcal{N}_{W}^{2}(0, T, X)$ the space of the predictable $X$-valued processes endowed with the norm $\|\phi\|_{\mathcal{N}_{W}^{2}(0, T, X)}^{2}:=$ $\mathbb{E}\left[\int_{0}^{T}\|\phi\|_{X}^{2} d t\right]$ (see Da Prato-ZABCZYK [2] p. 94). 
Note that, once $h=0$, we recover exactly the choice used for deterministic damage models (rate-dependent), like, for example, in $[15,16,19]$.

Neglecting acceleration effects, the generalized principle of virtual powers (in the case when no macroscopic displacements are considered) leads to the balance law:

$$
B-\operatorname{div} \mathbf{H}=f \text {, }
$$

supplemented by a natural boundary condition $\mathbf{H} \cdot \mathbf{n}=0 . B$ and $\mathbf{H}$ are microscopic forces and stresses acting in the material specified by the following constitutive laws:

$$
B=\frac{\partial \Psi}{\partial u}+\frac{\partial \Phi}{\partial z_{t}}, \quad \mathbf{H}=\frac{\partial \Psi}{\partial \nabla u} .
$$

Hence, (1.1a) and (1.1c) easily follow combining (1.2)-(1.3) with (1.4)-(1.5).

\subsection{Goal of the study and outline of the paper}

First of all, let us introduce the concept of solution we are interested in for Problem (1.1).

Definition 1

Any pair $(u, \xi)$ with $u$ belonging to $\mathcal{N}_{w}^{2}\left(0, T, H^{1}(D)\right) \cap L^{\infty}\left(0, T, L^{2}\left(\Omega, H^{1}(D)\right)\right) \cap L^{2}\left(\Omega, \mathscr{C}\left(0, T ; L^{2}(D)\right)\right), 0 \leq u \leq 1$ and $\xi$ in $\mathcal{N}_{w}^{2}\left(0, T, L^{2}(D)\right)$ is a solution to the stochastic problem (1.1) if almost everywhere in $(0, T), \mathbb{P}$-almost surely in $\Omega$, and for any $v$ in $V$

$$
\int_{D} \partial_{t}\left(u-\int_{0}^{\cdot} h(u) d W(s)\right) v d x+\int_{D} \nabla u . \nabla v d x+\int_{D} \xi v d x=\int_{D}\left(w_{s}(u)+f\right) v d x
$$

with $u(., 0)=u_{0}$ and $\xi \in \partial I_{[0,1]}(u)$.

Remark 1 (Sense of the initial condition)

Because $u \in L^{2}\left(\Omega, \mathscr{C}\left(0, T ; L^{2}(D)\right)\right)$, it satisfies the initial condition in the following sense:

$$
\mathbb{P} \text {-a.s in } \Omega, u(., 0)=\lim _{t \rightarrow 0} u(., t) \text { in } L^{2}(D) \text {. }
$$

The aim of this paper is to show the following result:

Theorem 1.2

Under hypotheses $H_{1}-H_{5}$, there exists a unique pair $(u, \xi)$ solution to Problem (1.1) in the sense of Definition 1.

To do this, we firstly consider in Section 2 a family of approximating problems (by regularization of the maximal monotone operator $\left.\partial l_{[0,1]}\right)$ depending on a parameter $\epsilon>0$, and we prove a related well-posedness result. Secondly, in Section 3 , we perform the passage to the limit with respect to the approximating parameter $\epsilon>0$, by compactness and monotonicity tools, and we show that the approximate solutions converge to a solution of Problem (1.1), in the sense of Definition 1, and that this solution is unique.

\section{Regularized problem}

Set $\epsilon>0$ and consider the following stochastic problem:

$$
\left\{\begin{aligned}
d u_{\epsilon}+\left(\psi_{\epsilon}\left(u_{\epsilon}\right)-\Delta u_{\epsilon}\right) d t & =\left(w_{s}\left(u_{\epsilon}\right)+f\right) d t+h\left(u_{\epsilon}\right) d W & & \text { in } \Omega \times D \times(0, T), \\
u_{\epsilon}(\omega, x, t=0) & =u_{0}(x), & & \omega \in \Omega, x \in D, \\
\nabla u_{\epsilon} . \mathbf{n} & =0 & & \text { in } \Omega \times \partial D \times(0, T),
\end{aligned}\right.
$$

where $\psi_{\epsilon}$ denotes the Yosida approximation of $\partial /_{[0,1]}$ (see, e.g., $\left.[1,20]\right)$. For all $v \in \mathbb{R}$, it holds

$$
\epsilon(v)=-\frac{(v)^{-}}{\epsilon}+\frac{(v-1)^{+}}{\epsilon}=\left\{\begin{array}{cl}
\frac{v}{\epsilon} & \text { if } v \leqslant 0 \\
0 & \text { if } v \in[0,1] \\
\frac{v-1}{\epsilon} & \text { if } v \geqslant 1
\end{array}\right.
$$

Definition 2

A function $u_{\epsilon} \in L^{2}\left(\Omega, \mathscr{C}\left(0, T ; L^{2}(D)\right)\right) \cap L^{\infty}\left(0, T ; L^{2}\left(\Omega, H^{1}(D)\right)\right) \cap \mathcal{N}_{w}^{2}\left(0, T, H^{1}(D)\right)$ such that $\partial_{t}\left(u_{\epsilon}-\int_{0} h\left(u_{\epsilon}\right) d W\right)$ and $\Delta u_{\epsilon}$ belong to $L^{2}(\Omega \times Q)$ is a solution to the stochastic problem (2.1) if almost everywhere in $(0, T)$ and $\mathbb{P}$-almost surely in $\Omega$, the following variational formulation holds for any $v \in H^{1}(D)$ :

$$
\int_{D} \partial_{t}\left(u_{\epsilon}-\int_{0} h\left(u_{\epsilon}\right) d W(s)\right) v d x+\int_{D} \nabla u_{\epsilon} \cdot \nabla v d x+\int_{D} \psi_{\epsilon}\left(u_{\epsilon}\right) v d x=\int_{D}\left(w_{s}\left(u_{\epsilon}\right)+f\right) v d x
$$

with $u_{\epsilon}(., 0)=u_{0}$. 
Remark 2

Because $u_{\epsilon} \in L^{2}\left(\Omega, \mathscr{C}\left(0, T ; L^{2}(D)\right)\right)$, it satisfies the initial condition in the sense of Remark 1.

We have the following well-posedness result:

Theorem 2.1

Under hypotheses $H_{1}-H_{5}$ and for any $\epsilon>0$, there exists a unique solution $u_{\epsilon}$ in the sense of Definition 2 to the stochastic Problem (2.1).

\subsection{Existence of $u_{\epsilon}$}

Note that the well-posedness of Problem (2.1) could be deduced from classic results in the literature [2, 21], but for a matter of selfcontainedness and to prepare the a priori estimates needed to pass to the limit on the penalization procedure, we propose to detail a result of existence of a solution based on an implicit time-discretization scheme for the deterministic part and an explicit one for the Itô part.

We consider a positive integer $N$ and denote by $\Delta t=\frac{T}{N}$ and $t_{n}=n \Delta t, n \in\{0, \ldots, N\}$. Let us first introduce some classical notations needed in the sequel.

Definition 3

For any sequence $\left(x_{n}\right) \subset X$, where $X$ is a separable Banach space, let us denote by the following:

$$
\begin{aligned}
x^{\Delta t} & =\sum_{k=0}^{N-1} x_{k+1} \mathbb{1}_{\left[t_{k}, t_{k+1}\right)} \\
\tilde{x}^{\Delta t} & =\sum_{k=0}^{N-1}\left[\frac{x_{k+1}-x_{k}}{\Delta t}\left(t-t_{k}\right)+x_{k}\right] \mathbb{1}_{\left[t_{k}, t_{k+1}\right)} \\
\frac{\partial \tilde{x}^{\Delta t}}{\partial t} & =\sum_{k=0}^{N-1} \frac{x_{k+1}-x_{k}}{\Delta t} \mathbb{1}_{\left[t_{k}, t_{k+1}\right)}
\end{aligned}
$$

and elementary calculations yield for an arbitrary constant $C>0$ independent of $\Delta t$ :

$$
\begin{aligned}
& \left\|x^{\Delta t}\right\|_{L^{2}(0, T ; X)}^{2}=\Delta t \sum_{k=1}^{N}\left\|x_{k}\right\|_{X}^{2} ;\left\|\tilde{x}^{\Delta t}\right\|_{L^{2}(0, T ; X)}^{2} \leqslant C \Delta t \sum_{k=0}^{N}\left\|x_{k}\right\|_{X^{\prime}}^{2} ; \\
& \left\|x^{\Delta t}-\tilde{x}^{\Delta t}\right\|_{L^{2}(0, T ; X)}^{2}=\Delta t \sum_{k=0}^{N-1}\left\|x_{k+1}-x_{k}\right\|_{X ;}^{2} \\
& \left\|\frac{\partial \tilde{x}^{\Delta t}}{\partial t}\right\|_{L^{2}(0, T ; X)}^{2}=\frac{1}{\Delta t} \sum_{k=0}^{N-1}\left\|x_{k+1}-x_{k}\right\|_{X^{\prime}}^{2} ; \\
& \left\|x^{\Delta t}\right\|_{L^{\infty}(0, T ; X)}=\max _{k=1, \ldots, N}\left\|x_{k}\right\|_{X} ; \quad\left\|\tilde{x}^{\Delta t}\right\|_{L^{\infty}(0, T ; X)}=\max _{k=0, . ., N}\left\|x_{k}\right\|_{X} .
\end{aligned}
$$

The discretization scheme is the following one: For given small positive parameters $\Delta t$ and $u_{n}$ in $L^{2}\left(\left(\Omega, \mathcal{F}_{t_{n}}\right) ; H^{1}(D)\right)(n \geq 0)$, we aim to find $u_{n+1}$ in $L^{2}\left(\left(\Omega, \mathcal{F}_{t_{n+1}}\right) ; H^{1}(D)\right)$, such that $\mathbb{P}$-a.s in $\Omega$ and for any $v$ in $H^{1}(D)$

$$
\begin{aligned}
& \int_{D}\left(u_{n+1}-u_{n}\right) v d x+\Delta t \int_{D}\left(\nabla u_{n+1} \cdot \nabla v+\psi_{\epsilon}\left(u_{n+1}\right) v\right) d x \\
= & \Delta t \int_{D}\left(w_{s}\left(u_{n+1}\right)+f_{n}\right) v d x+\left(W_{n+1}-W_{n}\right) \int_{D} h\left(u_{n}\right) v d x,
\end{aligned}
$$

where $W_{n}=W\left(t_{n}\right)$ and $f_{n}=\frac{1}{\Delta t} \int_{t_{n-1}}^{t_{n}} f(s) d s^{\S}$. To proceed in this way, we prove that, once $n$ is fixed, we can find the solution for the step $n+1$ by a fixed point argument, once it is ensured that the time step of the discrete scheme is sufficiently small.

Lemma 1

Set $u_{n} \in L^{2}\left(\left(\Omega, \mathcal{F}_{t_{n}}\right) ; H^{1}(D)\right)$. If $\Delta t<\frac{1}{\left(L_{s}+\frac{1}{\epsilon}\right)}$, there exists a unique $u_{n+1} \in L^{2}\left(\left(\Omega, \mathcal{F}_{t_{n+1}}\right) ; H^{1}(D)\right)$, satisfying the variational problem (2.2).

Proof

Consider $\mathbb{T}$ the application defined by the following:

$$
\begin{aligned}
\mathbb{T}: L^{2}\left(\left(\Omega, \mathcal{F}_{t_{n+1}}\right) ; L^{2}(D)\right) & \rightarrow L^{2}\left(\left(\Omega, \mathcal{F}_{t_{n+1}}\right) ; H^{1}(D)\right) \\
S & \mapsto \mathbb{T}(S),
\end{aligned}
$$

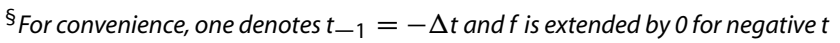


where for any $S \in L^{2}\left(\left(\Omega, \mathcal{F}_{t_{n+1}}\right) ; L^{2}(D)\right), u=\mathbb{T}(S)$ is the solution in $L^{2}\left(\left(\Omega, \mathcal{F}_{t_{n+1}}\right) ; H^{1}(D)\right)$ of the variational problem:

$$
\begin{aligned}
& \mathbb{E}\left[\int_{D}\left(u-u_{n}\right) v d x\right]+\Delta t \mathbb{E}\left[\int_{D} \nabla u \cdot \nabla v+\psi_{\epsilon}(S) v d x\right] \\
= & \Delta t \mathbb{E}\left[\int_{D}\left(w_{s}(S)+f_{n}\right) v d x\right]+\mathbb{E}\left[\left(W_{n+1}-W_{n}\right) \int_{D} h\left(u_{n}\right) v d x\right], \forall v \in L^{2}\left(\left(\Omega, \mathcal{F}_{t_{n+1}}\right) ; H^{1}(D)\right) .
\end{aligned}
$$

By denoting $B=u_{n}+\Delta t\left(w_{s}(S)+f_{n}-\psi_{\epsilon}(S)\right)+\left(W_{n+1}-W_{n}\right) h\left(u_{n}\right) \in L^{2}\left(\left(\Omega, \mathcal{F}_{t_{n+1}}\right) ; L^{2}(D)\right)$, this variational problem can be rewritten as follows:

$$
\mathbb{E}\left[\int_{D} u v+\Delta t \nabla u . \nabla v d x\right]=\mathbb{E}\left[\int_{D} B v d x\right], \forall v \in L^{2}\left(\left(\Omega, \mathcal{F}_{t_{n+1}}\right) ; H^{1}(D)\right) .
$$

Thanks to the theorem of Lax-Milgram, $\mathbb{T}$ is a well-defined function. Moreover, for any $S_{1}, S_{2} \in L^{2}\left(\left(\Omega, \mathcal{F}_{t_{n+1}}\right) ; L^{2}(D)\right)$, by denoting $u_{1}=\mathbb{T}\left(S_{1}\right)$ and $u_{2}=\mathbb{T}\left(S_{2}\right)$, one has that for any $v$ in $L^{2}\left(\left(\Omega, \mathcal{F}_{t_{n+1}}\right) ; H^{1}(D)\right)$ :

$$
\mathbb{E}\left[\int_{D}\left(u_{1}-u_{2}\right) v+\Delta t \nabla\left(u_{1}-u_{2}\right) \cdot \nabla v d x\right]=\mathbb{E}\left[\int_{D}\left(B_{1}-B_{2}\right) v d x\right]
$$

where

$$
B_{1}=u_{n}+\Delta t\left(w_{s}\left(S_{1}\right)+f_{n}-\psi_{\epsilon}\left(S_{1}\right)\right)+\left(W_{n+1}-W_{n}\right) h\left(u_{n}\right),
$$

and

$$
B_{2}=u_{n}+\Delta t\left(w_{s}\left(S_{2}\right)+f_{n}-\psi_{\epsilon}\left(S_{2}\right)\right)+\left(W_{n+1}-W_{n}\right) h\left(u_{n}\right) .
$$

Then, for $v=u_{1}-u_{2}$, we have

$$
\begin{aligned}
\mathbb{E}\left[\int_{D}\left|u_{1}-u_{2}\right|^{2} d x\right] & \leq \mathbb{E}\left[\int_{D}\left|u_{1}-u_{2}\right|^{2}+\Delta t\left|\nabla\left(u_{1}-u_{2}\right)\right|^{2} d x\right] \\
& =\Delta t \mathbb{E}\left[\int_{D}\left(w_{s}\left(S_{1}\right)-w_{s}\left(S_{2}\right)+\psi_{\epsilon}\left(S_{2}\right)-\psi_{\epsilon}\left(S_{1}\right)\right)\left(u_{1}-u_{2}\right) d x\right] \\
& \leqslant \Delta t\left(L_{s}+\frac{1}{\epsilon}\right) \mathbb{E}\left[\int_{D}\left|u_{1}-u_{2}\right|\left|S_{1}-S_{2}\right| d x\right]
\end{aligned}
$$

which yields

$$
\left\|u_{1}-u_{2}\right\|_{L^{2}\left(\left(\Omega, \mathcal{F}_{t_{n+1}}\right) ; L^{2}(D)\right)} \leqslant \Delta t\left(L_{s}+\frac{1}{\epsilon}\right)\left\|S_{1}-S_{2}\right\|_{L^{2}\left(\left(\Omega, \mathcal{F}_{t_{n+1}}\right) ; L^{2}(D)\right)} .
$$

Then, for $\Delta t<\frac{1}{\left(L_{s}+\frac{1}{\epsilon}\right)}, \mathbb{T}$ is a contractive mapping in $L^{2}\left(\left(\Omega, \mathcal{F}_{t_{n+1}}\right) ; L^{2}(D)\right)$, and the result holds.

Proposition 2.2 (Estimates on the approximate sequences)

There exists a constant $C>0$ that only depends on $T, C_{h}, u_{0}, L_{s}$, and $f$ such that

$$
\begin{aligned}
\left\|u^{\Delta t}\right\|_{L^{\infty}\left(0, T ; L^{2}(\Omega \times D)\right)},\left\|\tilde{u}^{\Delta t}\right\|_{L^{\infty}\left(0, T ; L^{2}(\Omega \times D)\right)} & \leqslant C \\
\left\|\nabla u^{\Delta t}\right\|_{L^{2}(\Omega \times Q)} & \leqslant C \\
\left\|u^{\Delta t}-\tilde{u}^{\Delta t}\right\|_{L^{2}(\Omega \times Q)} & \leqslant C \Delta t .
\end{aligned}
$$

Proof

By using the test function $v=u_{n+1}$ in (2.2), we have

$$
\begin{aligned}
& \int_{D}\left(u_{n+1}-u_{n}\right) u_{n+1} d x+\Delta t \int_{D}\left|\nabla u_{n+1}\right|^{2}+\psi_{\epsilon}\left(u_{n+1}\right) u_{n+1} d x \\
= & \Delta t \int_{D}\left(w_{s}\left(u_{n+1}\right)+f_{n}\right) u_{n+1} d x+\left(W_{n+1}-W_{n}\right) \int_{D} h\left(u_{n}\right) u_{n+1} d x .
\end{aligned}
$$

Using the formula $a b=\frac{1}{2}\left[a^{2}+b^{2}-(a-b)^{2}\right]$ with $a=u_{n+1}-u_{n}$ and $b=u_{n+1}$ yields

$$
\begin{aligned}
& \frac{1}{2} \int_{D}\left|u_{n+1}-u_{n}\right|^{2} d x+\frac{1}{2} \int_{D}\left|u_{n+1}\right|^{2} d x-\frac{1}{2} \int_{D}\left|u_{n}\right|^{2} d x+\Delta t \int_{D}\left|\nabla u_{n+1}\right|^{2}+\psi_{\epsilon}\left(u_{n+1}\right) u_{n+1} d x \\
= & \Delta t \int_{D}\left(w_{s}\left(u_{n+1}\right)+f_{n}\right) u_{n+1} d x+\left(W_{n+1}-W_{n}\right) \int_{D} h\left(u_{n}\right) u_{n} d x \\
& +\left(W_{n+1}-W_{n}\right) \int_{D} h\left(u_{n}\right)\left(u_{n+1}-u_{n}\right) d x .
\end{aligned}
$$


Thanks to the monotonicity of $\psi_{\epsilon}$ and the fact that $\psi_{\epsilon}(0)=0$, we get for any $\delta>0$

$$
\begin{aligned}
& \frac{1}{2} \int_{D}\left|u_{n+1}-u_{n}\right|^{2} d x+\frac{1}{2} \int_{D}\left|u_{n+1}\right|^{2} d x-\frac{1}{2} \int_{D}\left|u_{n}\right|^{2} d x+\Delta t \int_{D}\left|\nabla u_{n+1}\right|^{2} d x \\
\leqslant & \Delta t \int_{D}\left(w_{s}\left(u_{n+1}\right)+f_{n}\right)\left(u_{n+1}-u_{n}+u_{n}\right) d x+\left(W_{n+1}-W_{n}\right) \int_{D} h\left(u_{n}\right) u_{n} d x \\
& +\frac{1}{2 \delta}\left(W_{n+1}-W_{n}\right)^{2} \int_{D} h^{2}\left(u_{n}\right) d x+\frac{\delta}{2} \int_{D}\left|u_{n+1}-u_{n}\right|^{2} d x .
\end{aligned}
$$

Then by taking the expectation, we get $\mathbb{E}\left[\left(W_{n+1}-W_{n}\right) \int_{D} h\left(u_{n}\right) u_{n} d x\right]=0$

$$
\begin{aligned}
& \frac{1}{2} \mathbb{E}\left[\int_{D}\left|u_{n+1}-u_{n}\right|^{2}+\left|u_{n+1}\right|^{2}-\left|u_{n}\right|^{2} d x\right]+\Delta t \mathbb{E}\left[\int_{D}\left|\nabla u_{n+1}\right|^{2} d x\right] \\
\leqslant & \frac{\Delta t}{2} \mathbb{E}\left[\int_{D} L_{s}^{2}\left|u_{n+1}\right|^{2}+\left|u_{n+1}-u_{n}\right|^{2}+L_{s}^{2}\left|u_{n+1}\right|^{2}+\left|u_{n}\right|^{2} d x\right] \\
& +\frac{\Delta t}{2} \mathbb{E}\left[\int_{D}\left|f_{n}\right|^{2}+\left|u_{n+1}-u_{n}\right|^{2}+\left|f_{n}\right|^{2}+\left|u_{n}\right|^{2} d x\right] \\
& +\frac{1}{2 \delta} \mathbb{E}\left[\left(W_{n+1}-W_{n}\right)^{2}\right] \mathbb{E}\left[\int_{D} h^{2}\left(u_{n}\right) d x\right]+\frac{\delta}{2} \mathbb{E}\left[\int_{D}\left|u_{n+1}-u_{n}\right|^{2} d x\right] .
\end{aligned}
$$

And, for example, for $\delta=\frac{1}{2}$,

$$
\begin{aligned}
& \frac{1}{4} \mathbb{E}\left[\int_{D}\left|u_{n+1}-u_{n}\right|^{2} d x\right]+\left(\frac{1}{2}-\Delta t L_{s}^{2}\right) \mathbb{E}\left[\int_{D}\left|u_{n+1}\right|^{2}-\left|u_{n}\right|^{2} d x\right]+\Delta t \mathbb{E}\left[\int_{D}\left|\nabla u_{n+1}\right|^{2} d x\right] \\
\leqslant & \Delta t \mathbb{E}\left[\int_{D}\left|u_{n+1}-u_{n}\right|^{2} d x\right]+\Delta t\left(1+L_{s}^{2}\right) \mathbb{E}\left[\int_{D}\left|u_{n}\right|^{2} d x\right]+\Delta t \mathbb{E}\left[\int_{D}\left|f_{n}\right|^{2} d x\right]+\Delta t \mathbb{E}\left[\int_{D} h^{2}\left(u_{n}\right) d x\right],
\end{aligned}
$$

where we used the fact that $\mathbb{E}\left[\left(W_{n+1}-W_{n}\right)^{2}\right]=\Delta t$.

Thus, for $\Delta t$ small enough, we can infer that

$$
\begin{aligned}
& \frac{1}{8} \mathbb{E}\left[\int_{D}\left|u_{n+1}-u_{n}\right|^{2} d x\right]+\frac{1}{4} \mathbb{E}\left[\int_{D}\left|u_{n+1}\right|^{2}-\left|u_{n}\right|^{2} d x\right]+\Delta t \mathbb{E}\left[\int_{D}\left|\nabla u_{n+1}\right|^{2} d x\right] \\
\leqslant & \Delta t\left(1+C_{h}^{2}+L_{s}^{2}\right) \mathbb{E}\left[\int_{D}\left|u_{n}\right|^{2} d x\right]+\Delta t \mathbb{E}\left[\int_{D}\left|f_{n}\right|^{2} d x\right] .
\end{aligned}
$$

Consequently, for any $k \in\{0, \ldots, N\}$ if one denotes by $\|$.$\| the norm in L^{2}(D)$

$$
\begin{aligned}
& \frac{1}{8} \sum_{n=0}^{k-1} \mathbb{E}\left[\left\|u_{n+1}-u_{n}\right\|^{2}\right]+\frac{1}{4} \mathbb{E}\left[\left\|u_{k}\right\|^{2}\right]+\Delta t \sum_{n=0}^{k-1} \mathbb{E}\left[\left\|\nabla u_{n+1}\right\|^{2}\right] \\
\leqslant & \frac{1}{4} \mathbb{E}\left[\left\|u_{0}\right\|^{2}\right]+\Delta t\left(1+C_{h}^{2}+L_{s}^{2}\right) \sum_{n=0}^{k-1} \mathbb{E}\left[\left\|u_{n}\right\|^{2}\right]+\Delta t \sum_{n=0}^{k-1} \mathbb{E}\left[\left\|f_{n}\right\|^{2}\right] .
\end{aligned}
$$

Note that

$$
\begin{aligned}
\Delta t \sum_{n=0}^{k-1} \mathbb{E}\left[\left\|f_{n}\right\|^{2}\right] & =\Delta t \sum_{n=0}^{k-1} \mathbb{E}\left[\left\|\frac{1}{\Delta t} \int_{t_{n-1}}^{t_{n}} f(s) d s\right\|^{2}\right] \\
& =\frac{1}{\Delta t} \sum_{n=0}^{k-1} \mathbb{E}\left[\int_{D}\left(\int_{t_{n-1}}^{t_{n}} f(s) d s\right)^{2} d x\right] \\
& \leqslant \frac{\Delta t}{\Delta t} \sum_{n=0}^{k-1} \mathbb{E}\left[\int_{D} \int_{t_{n-1}}^{t_{n}}|f(s)|^{2} d s d x\right] \\
& \leqslant\|f\|_{L^{2}(\Omega \times Q)}^{2}
\end{aligned}
$$

The discrete Gronwall lemma asserts then that by denoting $\tilde{C}=4\|f\|_{L^{2}(\Omega \times Q)}^{2}+\left\|u_{0}\right\|_{L^{2}(D)}^{2}$

$$
\left.\mathbb{E}\left[\left\|u_{k}\right\|^{2}\right] \leqslant \tilde{C} \exp \sum_{n=0}^{k-1} 4 \Delta t\left(1+C_{h}^{2}+L_{s}^{2}\right)\right) \leqslant \tilde{C} e^{4 T\left(1+C_{h}^{2}+L_{s}^{2}\right)} .
$$


In this way,

$$
\begin{aligned}
& \frac{1}{4} \mathbb{E}\left[\left\|u_{k}\right\|^{2}\right]+\frac{1}{8} \sum_{n=0}^{k-1} \mathbb{E}\left[\left\|u_{n+1}-u_{n}\right\|^{2}\right]+\Delta t \sum_{n=0}^{k-1} \mathbb{E}\left[\left\|\nabla u_{n+1}\right\|^{2}\right] \\
\leqslant & \frac{1}{4}\left\|u_{0}\right\|^{2}+\|f\|_{L^{2}(\Omega \times Q)}^{2}+\tilde{C} T\left(1+C_{h}^{2}+L_{s}^{2}\right) e^{4 T\left(1+C_{h}^{2}+L_{s}^{2}\right)} .
\end{aligned}
$$

Using the notations of Definition 3 , we conclude that there exists a constant $C>0$ only depending on $T, C_{h}, u_{0}, L_{s}$, and $f$ such that

$$
\begin{aligned}
\left\|u^{\Delta t}\right\|_{L^{\infty}\left(0, T ; L^{2}(\Omega \times D)\right)},\left\|\tilde{u}^{\Delta t}\right\|_{L^{\infty}\left(0, T ; L^{2}(\Omega \times D)\right)} & \leqslant C \\
\left\|\nabla u^{\Delta t}\right\|_{L^{2}(\Omega \times Q)} & \leqslant C \\
\left\|u^{\Delta t}-\tilde{u}^{\Delta t}\right\|_{L^{2}(\Omega \times Q)} & \leqslant C \sqrt{\Delta t},
\end{aligned}
$$

which concludes the proof.

Proposition 2.3 (Additional estimates)

There exists a constant $C>0$ that only depends on $T, C_{h}, u_{0}, L_{s}$, and $f$ and such that

$$
\begin{aligned}
\left\|u^{\Delta t}\right\|_{L^{\infty}\left(0, T ; L^{2}\left(\Omega ; H^{1}(D)\right)\right)},\left\|\tilde{u}^{\Delta t}\right\|_{L^{\infty}\left(0, T ; L^{2}\left(\Omega ; H^{1}(D)\right)\right)} & \leqslant C\left(1+\left\|\psi_{\epsilon}\left(u^{\Delta t}\right)\right\|_{L^{2}(\Omega \times Q)}\right) \\
\left\|\tilde{B}^{\Delta t}\right\|_{L^{2}\left(\Omega \times(0, T) ; H^{1}(D)\right)},\left\|\partial_{t}\left(\tilde{u}^{\Delta t}-\tilde{B}^{\Delta t}\right)\right\|_{L^{2}(\Omega \times Q)} & \leqslant C\left(1+\left\|\psi_{\epsilon}\left(u^{\Delta t}\right)\right\|_{L^{2}(\Omega \times Q)}\right), \\
\left\|\tilde{u}^{\Delta t}-u^{\Delta t}\right\|_{L^{2}\left(0, T ; L^{2}\left(\Omega ; H^{1}(D)\right)\right)} & \leqslant C\left(1+\left\|\psi_{\epsilon}\left(u^{\Delta t}\right)\right\|_{L^{2}(\Omega \times Q)}\right) \sqrt{\Delta t},
\end{aligned}
$$

where $\tilde{B}^{\Delta t}$ is given by Definition 3 with $B_{n}=\int_{0}^{t_{n}} h\left(u^{\Delta t}(s-\Delta t)\right) d W(s), n \in\{0, \ldots, N\}$.

Proof

By denoting as previously the norm in $L^{2}(D)$ by $\|$.$\| , setting the test function v=u_{n+1}-u_{n}-\left(W_{n+1}-W_{n}\right) h\left(u_{n}\right)$ in $(2.2)$ yields for any $\delta>0$, after taking the expectation:

$$
\begin{aligned}
\mathbb{E} & {\left[\|v\|^{2}\right]-\delta \frac{\Delta t^{2}}{2} \mathbb{E}\left[\left\|\psi_{\epsilon}\left(u_{n+1}\right)\right\|^{2}\right]-\frac{1}{2 \delta} \mathbb{E}\left[\|v\|^{2}\right] } \\
& +\frac{\Delta t}{2} \mathbb{E}\left[\left\|\nabla u_{n+1}\right\|^{2}+\frac{1}{2}\left\|\nabla u_{n+1}-\nabla u_{n}\right\|^{2}-\left\|\nabla u_{n}\right\|^{2}-2 \Delta t\left\|\nabla h\left(u_{n}\right)\right\|^{2}\right] \\
\leqslant & \mathbb{E}\left[\|v\|^{2}\right]+\Delta t \mathbb{E}\left[\int_{D} \psi_{\epsilon}\left(u_{n+1}\right) v d x\right]+\Delta t \mathbb{E}\left[\int_{D} \nabla u_{n+1} . \nabla v d x\right] \\
= & \Delta t \mathbb{E}\left[\int_{D}\left(w_{s}\left(u_{n+1}\right)+f_{n}\right) v d x\right] \\
\leqslant & \delta \frac{\Delta t^{2}}{2}\left(L_{s}^{2} \mathbb{E}\left[\left\|u_{n+1}\right\|^{2}\right]+\mathbb{E}\left[\left\|f_{n}\right\|^{2}\right]\right)+\frac{1}{\delta} \mathbb{E}\left[\|v\|^{2}\right] .
\end{aligned}
$$

Indeed, because $\mathbb{E}\left[\left(W_{n+1}-W_{n}\right) \int_{D} \nabla u_{n} . \nabla h\left(u_{n}\right) d x\right]=0$ and $\mathbb{E}\left[\left(W_{n+1}-W_{n}\right)^{2}\right]=\Delta t$, we have

$$
\begin{aligned}
\mathbb{E}\left[\int_{D} \nabla u_{n+1} \cdot \nabla v d x\right]= & \frac{1}{2}\left(\mathbb{E}\left[\left\|\nabla u_{n+1}\right\|^{2}\right]+\mathbb{E}\left[\left\|\nabla\left(u_{n+1}-u_{n}\right)\right\|^{2}\right]-\mathbb{E}\left[\left\|\nabla u_{n}\right\|^{2}\right]\right) \\
& -\mathbb{E}\left[\left(W_{n+1}-W_{n}\right) \int_{D} \nabla\left(u_{n+1}-u_{n}\right) . \nabla h\left(u_{n}\right) d x\right] \\
\geqslant & \frac{1}{2}\left(\mathbb{E}\left[\left\|\nabla u_{n+1}\right\|^{2}\right]+\frac{1}{2} \mathbb{E}\left[\left\|\nabla\left(u_{n+1}-u_{n}\right)\right\|^{2}\right]-\mathbb{E}\left[\left\|\nabla u_{n}\right\|^{2}\right]-2 \Delta t \mathbb{E}\left[\left\|\nabla h\left(u_{n}\right)\right\|^{2}\right]\right)
\end{aligned}
$$

Finally, for $\delta=3$, one gets

$$
\begin{aligned}
& \frac{1}{2} \mathbb{E}\left[\|v\|^{2}\right]+\frac{\Delta t}{2} \mathbb{E}\left[\left\|\nabla u_{n+1}\right\|^{2}+\frac{1}{2}\left\|\nabla u_{n+1}-\nabla u_{n}\right\|^{2}-\left\|\nabla u_{n}\right\|^{2}\right] \\
\leqslant & 3 \frac{\Delta t^{2}}{2}\left(\mathbb{E}\left[\left\|\psi_{\epsilon}\left(u_{n+1}\right)\right\|^{2}\right]+L_{s}^{2} \mathbb{E}\left[\left\|u_{n+1}\right\|^{2}\right]\right)+3 \frac{\Delta t^{2}}{2} \mathbb{E}\left[\left\|f_{n}\right\|^{2}\right]+\Delta t^{2} C_{h}^{2} \mathbb{E}\left[\left\|\nabla u_{n}\right\|^{2}\right] .
\end{aligned}
$$


Then, for any $k \in\{0, \ldots, N\}$,

$$
\begin{aligned}
& \sum_{n=0}^{k-1} \mathbb{E}\left[\|v\|^{2}\right]+\Delta t \mathbb{E}\left[\left\|\nabla u_{k}\right\|^{2}\right]+\frac{\Delta t}{2} \sum_{n=0}^{k-1} \mathbb{E}\left[\left\|\nabla u_{n+1}-\nabla u_{n}\right\|^{2}\right] \\
\leqslant & \left.\Delta t \mid\left\|\nabla u_{0}\right\|^{2}+3 \Delta t^{2} \sum_{n=0}^{k-1} \mathbb{E}\left[\left\|\psi_{\epsilon}\left(u_{n+1}\right)\right\|^{2}\right]+L_{s}^{2} \mathbb{E}\left[\left\|u_{n+1}\right\|^{2}\right]\right)+3 \Delta t^{2} \sum_{n=0}^{k-1} \mathbb{E}\left[\left\|f_{n}\right\|^{2}\right]+2 \Delta t^{2} C_{h}^{2} \sum_{n=0}^{k-1} \mathbb{E}\left[\left\|\nabla u_{n}\right\|^{2}\right],
\end{aligned}
$$

and so

$$
\begin{aligned}
& \sum_{n=0}^{k-1} \Delta t \mathbb{E}\left[\left\|\frac{v}{\Delta t}\right\|^{2}\right]+\mathbb{E}\left[\left\|\nabla u_{k}\right\|^{2}\right]+\frac{1}{2} \sum_{n=0}^{k-1} \mathbb{E}\left[\left\|\nabla u_{n+1}-\nabla u_{n}\right\|^{2}\right] \\
\leqslant & \left.\left\|\nabla u_{0}\right\|^{2}+3 \Delta t \sum_{n=0}^{k-1} \mathbb{E}\left[\left\|\psi_{\epsilon}\left(u_{n+1}\right)\right\|^{2}\right]+L_{s}^{2} \mathbb{E}\left[\left\|u_{n+1}\right\|^{2}\right]\right)+3 \Delta t \sum_{n=0}^{k-1} \mathbb{E}\left[\left\|f_{n}\right\|^{2}\right]+2 \Delta t C_{h}^{2} \sum_{n=0}^{k-1} \mathbb{E}\left[\left\|\nabla u_{n}\right\|^{2}\right] \\
\leqslant & \left(1+2 C_{h}^{2}\right)\left\|\nabla u_{0}\right\|^{2}+3\left(\left\|\psi_{\epsilon}\left(u^{\Delta t}\right)\right\|_{L^{2}(\Omega \times Q)}^{2}+L_{s}^{2}\left\|u^{\Delta t}\right\|_{L^{2}(\Omega \times Q)}^{2}\right)+3\|f\|_{L^{2}(\Omega \times Q)}^{2}+2 C_{h}^{2}\left\|\nabla u^{\Delta t}\right\|_{L^{2}(\Omega \times Q)}^{2} .
\end{aligned}
$$

Using estimates given by Proposition 2.2, we deduce by denoting

$$
\begin{aligned}
\tilde{B}^{\Delta t} & =\sum_{k=0}^{N-1}\left(\frac{B_{k+1}-B_{k}}{\Delta t}\left(.-t_{k}\right)+B_{k}\right) \mathbb{1}_{\left[t_{k}, t_{k+1}\right)} \\
\text { with } B_{k} & =\int_{0}^{t_{k}} h\left(u^{\Delta t}(s-\Delta t)\right) d W(s)=\sum_{n=0}^{k-1}\left(W_{n+1}-W_{n}\right) h\left(u_{n}\right), k \in\{0, \ldots, N\},
\end{aligned}
$$

that $u^{\Delta t}$ and $\tilde{u}^{\Delta t}$ are bounded in $L^{\infty}\left(0, T ; L^{2}\left(\Omega ; H^{1}(D)\right)\right)$, that $\partial_{t}\left(\tilde{u}^{\Delta t}-\tilde{B}^{\Delta t}\right)$ is bounded in $L^{2}(\Omega \times Q)$, and that $\tilde{u}^{\Delta t}-u^{\Delta t} \rightarrow 0$ in $L^{2}\left(0, T ; L^{2}\left(\Omega ; H^{1}(D)\right)\right)$ as $\Delta t \rightarrow 0$.

Because, for any $k \in\{0, \ldots, N-1\}, E\left[\left\|\sum_{n=0}^{k-1}\left(W_{n+1}-W_{n}\right) h\left(u_{n}\right)\right\|^{2}\right]=\Delta t \sum_{n=0}^{k-1} E\left[\left\|h\left(u_{n}\right)\right\|^{2}\right]$, by Itô's isometry ([21] Proposition 2.3.5 p. 25), one gets

$$
\begin{aligned}
E\left[\int_{0}^{T}\left\|\tilde{B}^{\Delta t}(t)\right\|^{2} d t\right] & =\sum_{k=0}^{N-1} \int_{t_{k}}^{t_{k+1}} E\left[\left\|\frac{B_{k+1}-B_{k}}{\Delta t}\left(t-t_{k}\right)+B_{k}\right\|^{2}\right] d t \\
& \leq C \sum_{k=0}^{N-1} \int_{t_{k}}^{t_{k+1}} E\left[\left\|B_{k+1}-B_{k}\right\|^{2}\right]+E\left[\left\|B_{k}\right\|^{2}\right] d t \\
& =C \sum_{k=0}^{N-1} \Delta t\left\{E\left[\left\|\left(W_{k+1}-W_{k}\right) h\left(u_{k}\right)\right\|^{2}\right]+E\left[\left\|\sum_{n=0}^{k-1}\left(W_{n+1}-W_{n}\right) h\left(u_{n}\right)\right\|^{2}\right]\right\} \\
& \leq C \sum_{k=0}^{N-1} \Delta t^{2} \sum_{n=0}^{k} E\left[\left\|h\left(u_{n}\right)\right\|^{2}\right] .
\end{aligned}
$$

Thus,

$$
E\left[\int_{0}^{T}\left\|\tilde{B}^{\Delta t}(t)\right\|^{2} d t\right] \leq C \Delta t \sum_{n=0}^{N-1} E\left[\left\|h\left(u_{n}\right)\right\|^{2}\right]
$$

is bounded, and the proposition is proved because the same argument holds with the $H^{1}$ norm.

Proposition 2.4

There exists $u_{\epsilon} \in L^{2}\left(\Omega, \mathscr{C}\left(0, T ; L^{2}(D)\right)\right) \cap L^{\infty}\left(0, T ; L^{2}\left(\Omega, H^{1}(D)\right)\right) \cap \mathcal{N}_{w}^{2}\left(0, T, H^{1}(D)\right), h_{\epsilon}$ and $\chi_{\epsilon}$ in $L^{2}\left(0, T ; L^{2}\left(\Omega, H^{1}(D)\right)\right)$ and $w_{\epsilon}$ in $L^{2}(\Omega \times Q)$ such that, up to subsequences denoted in the same way, as $\Delta t \rightarrow 0$ (i.e., $\left.N \rightarrow+\infty\right)$,

$$
\begin{aligned}
u^{\Delta t}, \tilde{u}^{\Delta t} & \stackrel{*}{\rightarrow} u_{\epsilon} \text { in } L^{\infty}\left(0, T ; L^{2}\left(\Omega, H^{1}(D)\right)\right), \\
h\left(u^{\Delta t}\right) & \rightarrow h_{\epsilon} \text { in } L^{2}\left(0, T ; L^{2}\left(\Omega, H^{1}(D)\right)\right), \\
\epsilon\left(u^{\Delta t}\right) & \rightarrow \chi_{\epsilon} \text { in } L^{2}\left(0, T ; L^{2}\left(\Omega, H^{1}(D)\right)\right), \\
w_{s}\left(u^{\Delta t}\right) & \rightarrow w_{\epsilon} \text { in } L^{2}(\Omega \times Q), \\
\tilde{u}^{\Delta t}-\tilde{B}^{\Delta t} & \rightarrow u_{\epsilon}-\int_{0} h_{\epsilon}(s) d W(s) \text { in } L^{2}\left(\Omega, H^{1}(Q)\right), \\
\forall t \in[0, T], \tilde{u}^{\Delta t}(t) & \rightarrow u_{\epsilon}(t) \text { in } L^{2}(\Omega \times D) .
\end{aligned}
$$


Proof

Note that thanks to the estimates given by Propositions 2.2 and 2.3 , and noticing that $\left\|\psi_{\epsilon}\left(u^{\Delta t}\right)\right\|_{L^{2}(\Omega \times Q)}$ is bounded with respect to the parameter $\Delta t$, by compactness, the existence of the weak limits $u_{\epsilon}, h_{\epsilon}, \chi_{\epsilon}$, and $w_{\epsilon}$ is immediate. We mention that $u_{\epsilon}$ is a $H^{1}(D)$-valued predictable process as a weak limit of the continuous and adapted process $\tilde{u}^{\Delta t}(.-\Delta t)$ belonging to the Hilbert space $\mathcal{N}_{w}^{2}\left(0, T, H^{1}(D)\right)$ equipped with the norm of $L^{2}\left((0, T) \times \Omega, H^{1}(D)\right)$.

Let us now focus on the weak convergence of $\tilde{u}^{\Delta t}-\tilde{B}^{\Delta t}$ towards $u_{\epsilon}-\int_{0} h_{\epsilon}(s) d W(s)$ in $L^{2}\left(\Omega, H^{1}(Q)\right)$.

Firstly, because $\tilde{u}^{\Delta t}-\tilde{B}^{\Delta t}, \nabla\left(\tilde{u}^{\Delta t}-\tilde{B}^{\Delta t}\right)$ and $\partial_{t}\left(\tilde{u}^{\Delta t}-\tilde{B}^{\Delta t}\right)$ are bounded in $L^{2}(\Omega \times Q)$, there exists $\zeta_{\epsilon}$ in $L^{2}\left(\Omega, H^{1}(Q)\right)$ such that, up to a subsequence,

$$
\tilde{u}^{\Delta t}-\tilde{B}^{\Delta t} \rightarrow \zeta_{\epsilon} \text { in } L^{2}\left(\Omega, H^{1}(Q)\right) .
$$

Secondly, we show later that $\tilde{B}^{\Delta t}$ converges weakly to $\int_{0} h_{\epsilon}(s) d W(s)$ in $\mathscr{C}\left(0, T ; L^{2}(\Omega \times D)\right)$. In this way,

$$
\tilde{u}^{\Delta t}-\tilde{B}^{\Delta t} \rightarrow u_{\epsilon}-\int_{0} h_{\epsilon}(s) d W(s) \text { in } L^{2}\left(0, T ; L^{2}(\Omega \times D)\right),
$$

and then $\zeta_{\epsilon}=u_{\epsilon}-\int_{0} h_{\epsilon}(s) d W(s)$. Indeed, for any $t \in\left[t_{n}, t_{n+1}\right)$, we have

$$
\begin{aligned}
& \mathbb{E}\left[\left\|\tilde{B}^{\Delta t}(t)-\int_{0}^{t} h\left(u^{\Delta t}(s-\Delta t)\right) d W(s)\right\|^{2}\right] \\
= & \mathbb{E}\left[\left\|\frac{t-t_{n}}{\Delta t} \int_{t_{n}}^{t_{n+1}} h\left(u^{\Delta t}(s-\Delta t)\right) d W(s)-\int_{t_{n}}^{t} h\left(u^{\Delta t}(s-\Delta t)\right) d W(s)\right\|^{2}\right] \\
= & \mathbb{E}\left[\left\|\left(W_{n+1}-W_{n}\right) h\left(u_{n}\right) \frac{t-t_{n}}{\Delta t}-\left(W(t)-W_{n}\right) h\left(u_{n}\right)\right\|^{2}\right] \\
= & \mathbb{E}\left[\left\|h\left(u_{n}\right)\right\|^{2}\right] \times\left\{\mathbb{E}\left[\left(W_{n+1}-W_{n}\right)^{2}\right]\left(\frac{t-t_{n}}{\Delta t}\right)^{2}+\mathbb{E}\left[\left(W(t)-W_{n}\right)^{2}\right]\right\} \\
& -2 \mathbb{E}\left[\left\|h\left(u_{n}\right)\right\|^{2}\left(W_{n+1}-W_{n}\right) \frac{t-t_{n}}{\Delta t}\left(W(t)-W_{n}\right)\right] \\
= & \mathbb{E}\left[\left\|h\left(u_{n}\right)\right\|^{2}\right] \times\left\{\frac{\left(t-t_{n}\right)^{2}}{\Delta t}+\left(t-t_{n}\right)-2 \frac{\left(t-t_{n}\right) 2}{\Delta t}\right\} \\
& -2 \mathbb{E}\left[\left\|h\left(u_{n}\right)\right\|^{2}\left(W_{n+1}-W(t)\right) \frac{t-t_{n}}{\Delta t}\left(W(t)-W_{n}\right)\right] \\
= & \mathbb{E}\left[\left\|h\left(u_{n}\right)\right\|^{2}\right] \times\left\{\left(t-t_{n}\right)-\frac{\left(t-t_{n}\right)^{2}}{\Delta t}\right\} \\
\leqslant & C \Delta t,
\end{aligned}
$$

by using again the fact that $\mathbb{E}\left[\left(W_{n+1}-W_{n}\right)^{2}\right]=\Delta t, \mathbb{E}\left[\left(W(t)-W_{n}\right)^{2}\right]=t-t_{n}$, and the independence between the increment $W_{n+1}-W(t)$ and any $\mathcal{F}_{t}$ - measurable process ([2] p. 90).

Moreover, $h\left(u^{\Delta t}(.-\Delta t)\right)$ converges weakly to $h_{\epsilon}$ in $L^{2}(\Omega \times Q)$, and because the stochastic integral

$$
\begin{aligned}
I: L^{2}(\Omega \times Q) & \rightarrow \mathscr{C}\left(0, T ; L^{2}\left(\Omega, L^{2}(D)\right)\right) \\
v & \mapsto I(v):(\omega, x, t) \mapsto \int_{0}^{t} v(\omega, x, s) d W(s),
\end{aligned}
$$

is linear and continuous, we thus have

$$
\int_{0} h\left(u^{\Delta t}(s-\Delta t)\right) d W(s) \rightarrow \int_{0} h_{\epsilon}(s) d W(s) \text { in } \mathscr{C}\left(0, T ; L^{2}\left(\Omega, L^{2}(D)\right)\right),
$$

and so

$$
\tilde{B}^{\Delta t} \rightarrow \int_{0} h_{\epsilon}(s) d W(s) \text { in } \mathscr{C}\left(0, T ; L^{2}\left(\Omega, L^{2}(D)\right)\right) .
$$

Finally, because $\tilde{u}^{\Delta t}-\tilde{B}^{\Delta t}$ converges weakly in $L^{2}\left(\Omega, H^{1}(Q)\right)^{\uparrow}$, it converges weakly in

$$
L^{2}\left(\Omega, \mathscr{C}\left(0, T ; L^{2}(D)\right)\right) \hookrightarrow \mathscr{C}\left(0, T ; L^{2}\left(\Omega, L^{2}(D)\right)\right),
$$

then for any $t \in[0, T], \tilde{u}^{\Delta t}(t) \rightarrow u_{\epsilon}(t)$ in $L^{2}(\Omega \times D)$. Now, by the property of the stochastic integral ([2] Theorem 4.12 p. 101), $\int_{0} h_{\epsilon}(s) d W(s) \in L^{2}\left(\Omega, \mathscr{C}\left(0, T ; L^{2}(D)\right)\right)$, which allows us to conclude that $u_{\epsilon} \in L^{2}\left(\Omega, \mathscr{C}\left(0, T ; L^{2}(D)\right)\right)$.

Remark 3

Note that for $t=0$, we have $\tilde{u}^{\Delta t}(0)=u_{0} \rightarrow u_{\epsilon}(0)$ in $L^{2}(\Omega \times D)$ and then $u_{0}=u_{\epsilon}(0)$ (in the sense of Remark 1).

"We remind that $H^{1}(Q) \hookrightarrow \mathscr{C}\left([0, T], L^{2}(D)\right)$ 
By denoting $f_{\Delta t}=\sum_{k=0}^{N-1} f_{k} \mathbb{1}_{\left[t_{k}, t_{k+1}[\right.}$, we have for any $v$ in $H^{1}(D)$ and $\mathbb{P}$ a-s. in $\Omega$

$$
\int_{D} \partial_{t}\left(\tilde{u}^{\Delta t}-\tilde{B}^{\Delta t}\right) v d x+\int_{D} \nabla u^{\Delta t} . \nabla v d x+\int_{D} \epsilon\left(u^{\Delta t}\right) v d x=\int_{D}\left(w_{s}\left(u^{\Delta t}\right)+f_{\Delta t}\right) v d x .
$$

Because $f_{\Delta t} \rightarrow f$ in $L^{2}(\Omega \times Q)$, at the limit one gets that, for any $v$ in $H^{1}(D), \alpha$ in $L^{2}(0, T)$ and any $\beta$ in $L^{2}(\Omega)$, the following variational formulation holds:

$$
\begin{aligned}
& \int_{\Omega \times Q} \partial_{t}\left(u_{\epsilon}-\int_{0} h_{\epsilon}(s) d W(s)\right) v \alpha \beta d x d t d \mathbb{P}+\int_{\Omega \times Q}\left(\nabla u_{\epsilon} \cdot \nabla v\right) \alpha \beta d x d t d \mathbb{P} \\
& +\int_{\Omega \times Q} \chi_{\epsilon} v \alpha \beta d x d t d \mathbb{P}=\int_{\Omega \times Q}\left(w_{\epsilon}+f\right) v \alpha \beta d x d t d \mathbb{P} .
\end{aligned}
$$

Because $H^{1}(D)$ is separable, one gets that almost everywhere in $(0, T), \mathbb{P}$-almost surely in $\Omega$, and for any $v$ in $H^{1}(D)$,

$$
\int_{D} \partial_{t}\left(u_{\epsilon}-\int_{0} h_{\epsilon}(s) d W(s)\right) v d x+\int_{D} \nabla u_{\epsilon} \cdot \nabla v d x+\int_{D} \chi_{\epsilon} v d x=\int_{D}\left(w_{\epsilon}+f\right) v d x
$$

Particularly, almost everywhere in $(0, T), \mathbb{P}$-almost surely in $\Omega$, one gets that

$$
\Delta u_{\epsilon}=\partial_{t}\left(u_{\epsilon}-\int_{0} h_{\epsilon}(s) d W(s)\right)+\chi_{\epsilon}-w_{\epsilon}-f,
$$

a priori in $V^{\prime}$, but then in $L^{2}(D)$ because $L^{2}(D)$ is the chosen pivot space. Thus, $\Delta u_{\epsilon} \in L^{2}(\Omega \times Q)$ and $u_{\epsilon}$ is an Itô process of the following form:

$$
d u_{\epsilon}+\left(\chi_{\epsilon}-\Delta u_{\epsilon}\right) d t=\left(w_{\epsilon}+f\right) d t+h_{\epsilon} d W
$$

Proposition 2.5

The following convergences hold strongly in $L^{2}(\Omega \times Q)$ :

$$
\begin{aligned}
u^{\Delta t} & \rightarrow u_{\epsilon}, \\
h\left(u^{\Delta t}\right) & \rightarrow h\left(u_{\epsilon}\right), \\
\epsilon\left(u^{\Delta t}\right) & \rightarrow \psi_{\epsilon}\left(u_{\epsilon}\right), \\
w_{s}\left(u^{\Delta t}\right) & \rightarrow w_{s}\left(u_{\epsilon}\right) .
\end{aligned}
$$

Proof

By considering as previously the test function $v=u_{n+1}$ in (2.2), we have for any $n>0$ after taking the expectation:

$$
\begin{aligned}
& \frac{1}{2} \mathbb{E}\left[\left\|u_{n+1}-u_{n}\right\|^{2}\right]+\frac{1}{2} \mathbb{E}\left[\left\|u_{n+1}\right\|^{2}\right]-\frac{1}{2} \mathbb{E}\left[\left\|u_{n}\right\|^{2}\right]+\Delta t \mathbb{E}\left[\left\|\nabla u_{n+1}\right\|^{2}\right] \\
& +\Delta t \mathbb{E}\left[\int_{D} \psi_{\epsilon}\left(u_{n+1}\right) u_{n+1} d x\right] \\
& \leqslant \Delta t \mathbb{E}\left[\int_{D}\left(w_{s}\left(u_{n+1}\right)+f_{n}\right) u_{n+1} d x\right]+\frac{1}{2} \mathbb{E}\left[\left\|u_{n+1}-u_{n}\right\|^{2}\right]+\frac{\Delta t}{2} \mathbb{E}\left[\left\|h\left(u_{n}\right)\right\|^{2}\right],
\end{aligned}
$$

which yields, after multiplying by $e^{-c n \Delta t}$, for any positive $c$

$$
\begin{aligned}
& e^{-c n \Delta t} \mathbb{E}\left[\left\|u_{n+1}\right\|^{2}\right]-e^{-c(n-1) \Delta t} \mathbb{E}\left[\left\|u_{n}\right\|^{2}\right]+2 \Delta t e^{-c n \Delta t} \mathbb{E}\left[\left\|\nabla u_{n+1}\right\|^{2}\right] \\
& +2 \Delta t e^{-c n \Delta t} \mathbb{E}\left[\int_{D} \psi_{\epsilon}\left(u_{n+1}\right) u_{n+1} d x\right] \\
& \leqslant \\
& 2 e^{-c n \Delta t} \Delta t \mathbb{E}\left[\int_{D}\left(w_{s}\left(u_{n+1}\right)+f_{n}\right) u_{n+1} d x\right]+\Delta t e^{-c n \Delta t} \mathbb{E}\left[\left\|h\left(u_{n}\right)\right\|^{2}\right] \\
& +\left(e^{-c n \Delta t}-e^{-c(n-1) \Delta t}\right) \mathbb{E}\left[\left\|u_{n}\right\|^{2}\right] .
\end{aligned}
$$

For any $k>0$, by summing this inequality from $n=0$ to $k$, one gets

$$
\begin{aligned}
& e^{-c k \Delta t} \mathbb{E}\left[\left\|u_{k+1}\right\|^{2}\right]+2 \sum_{n=0}^{k} \Delta t e^{-c n \Delta t} \mathbb{E}\left[\left\|\nabla u_{n+1}\right\|^{2}\right]+2 \sum_{n=0}^{k} \Delta t e^{-c n \Delta t} \mathbb{E}\left[\int_{D} \psi_{\epsilon}\left(u_{n+1}\right) u_{n+1} d x\right] \\
& \leqslant e^{c \Delta t}\left\|u_{0}\right\|^{2}+2 \sum_{n=0}^{k} e^{-c n \Delta t} \Delta t \mathbb{E}\left[\int_{D}\left(w_{s}\left(u_{n+1}\right)+f_{n}\right) u_{n+1} d x\right]+\sum_{n=0}^{k} \Delta t e^{-c n \Delta t} \mathbb{E}\left[\left\|h\left(u_{n}\right)\right\|^{2}\right] \\
& +\sum_{n=0}^{k}\left(e^{-c n \Delta t}-e^{-c(n-1) \Delta t}\right) \mathbb{E}\left[\left\|u_{n}\right\|^{2}\right] .
\end{aligned}
$$


Because $\psi_{\epsilon}$ is non-decreasing and satisfies $\psi_{\epsilon}(0)=0$, we have

$$
\begin{aligned}
\mathbb{E}\left[\Delta t \sum_{n=0}^{k} e^{-c n \Delta t} \int_{D} \psi_{\epsilon}\left(u_{n+1}\right) u_{n+1} d x\right] & \geqslant \mathbb{E}\left[\sum_{n=0}^{k} \int_{t_{n}}^{t_{n+1}} e^{-c s} \int_{D} \psi_{\epsilon}\left(u_{n+1}\right) u_{n+1} d x d s\right] \\
& =\mathbb{E}\left[\int_{0}^{t_{k+1}} e^{-c s} \int_{D} \epsilon\left(u^{\Delta t}\right) u^{\Delta t} d x d s\right]
\end{aligned}
$$

and in the same way,

$$
\sum_{n=0}^{k} \Delta t e^{-c n \Delta t} \mathbb{E}\left[\left\|\nabla u_{n+1}\right\|^{2}\right] \geqslant \int_{0}^{t_{k+1}} e^{-c s} \mathbb{E}\left[\left\|\nabla u^{\Delta t}\right\|^{2}\right] d s
$$

Moreover,

$$
\begin{aligned}
\sum_{n=0}^{k} \Delta t e^{-c n \Delta t} \mathbb{E}\left[\left\|h\left(u_{n}\right)\right\|^{2}\right] & =\Delta t\left\|h\left(u_{0}\right)\right\|^{2}+\sum_{n=0}^{k-1} \Delta t e^{-c(n+1) \Delta t} \mathbb{E}\left[\left\|h\left(u_{n+1}\right)\right\|^{2}\right] \\
& \leqslant \Delta t\left\|h\left(u_{0}\right)\right\|^{2}+\sum_{n=0}^{k-1} \int_{t_{n}}^{t_{n+1}} e^{-c s} \mathbb{E}\left[\left\|h\left(u_{n+1}\right)\right\|^{2}\right] d s \\
& =\Delta t\left\|h\left(u_{0}\right)\right\|^{2}+\int_{0}^{t_{k}} e^{-c s} \mathbb{E}\left[\left\|h\left(u^{\Delta t}\right)\right\|^{2}\right] d s .
\end{aligned}
$$

Besides,

$$
\begin{aligned}
\sum_{n=0}^{k}\left(e^{-c n \Delta t}-e^{-c(n-1) \Delta t}\right) \mathbb{E}\left[\left\|u_{n}\right\|^{2}\right] & =\left(1-e^{c \Delta t}\right)\left\|u_{0}\right\|^{2}+\sum_{n=1}^{k}\left(e^{-c n \Delta t}-e^{-c(n-1) \Delta t}\right) \mathbb{E}\left[\left\|u_{n}\right\|^{2}\right] \\
& =\left(1-e^{c \Delta t}\right)\left\|u_{0}\right\|^{2}-c \sum_{n=1}^{k}\left(\int_{t_{n-1}}^{t_{n}} e^{-c s} d s\right) \mathbb{E}\left[\left\|u_{n}\right\|^{2}\right] \\
& =\left(1-e^{c \Delta t}\right)\left\|u_{0}\right\|^{2}-c \sum_{n=1}^{k}\left(\int_{t_{n-1}}^{t_{n}} e^{-c s} \mathbb{E}\left[\left\|u^{\Delta t}\right\|^{2}\right] d s\right) \\
& \leqslant\left(1-e^{c \Delta t}\right)\left\|u_{0}\right\|^{2}-c e^{-c \Delta t} \int_{0}^{t_{k}} e^{-c s} \mathbb{E}\left[\left\|u^{\Delta t}\right\|^{2}\right] d s .
\end{aligned}
$$

In this way, we can write inequality (2.4) in the following manner:

$$
\begin{aligned}
& e^{-c k \Delta t} \mathbb{E}\left[\left\|u_{k+1}\right\|^{2}\right]+2 \int_{0}^{t_{k+1}} e^{-c s} \mathbb{E}\left[\left\|\nabla u^{\Delta t}\right\|^{2}\right] d s+2 \int_{0}^{t_{k+1}} e^{-c s} \mathbb{E}\left[\int_{D} \epsilon\left(u^{\Delta t}\right) u^{\Delta t} d x\right] d s \\
& \leqslant e^{c \Delta t}\left\|u_{0}\right\|^{2}+2 \sum_{n=0}^{k} e^{-c n \Delta t} \Delta t \mathbb{E}\left[\int_{D}\left(w_{s}\left(u_{n+1}\right)+f_{n}\right) u_{n+1} d x\right] \\
& +\Delta t\left\|h\left(u_{0}\right)\right\|^{2}+\int_{0}^{t_{k}} e^{-c s} \mathbb{E}\left[\left\|h\left(u^{\Delta t}\right)\right\|^{2}\right] d s \\
& +\left(1-e^{c \Delta t}\right)\left\|u_{0}\right\|^{2}-c e^{-c \Delta t} \int_{0}^{t_{k}} e^{-c s} \mathbb{E}\left[\left\|u^{\Delta t}\right\|^{2}\right] d s .
\end{aligned}
$$

For $t \in\left[t_{k}, t_{k+1}\right)$, we have $e^{-c k \Delta t} \geqslant e^{-c t}$ and $(t-\Delta t)^{+} \leqslant t_{k}$ :

$$
\begin{aligned}
& e^{-c t} \mathbb{E}\left[\left\|u^{\Delta t}(t)\right\|^{2}\right]+2 \int_{0}^{t} e^{-c s} \mathbb{E}\left[\left\|\nabla u^{\Delta t}\right\|^{2}\right] d s+2 \int_{0}^{t} e^{-c s} \mathbb{E}\left[\int_{D} \epsilon\left(u^{\Delta t}\right) u^{\Delta t} d x\right] d s \\
& \leqslant\left\|u_{0}\right\|^{2}+2 \sum_{n=0}^{k} e^{-c n \Delta t} \Delta t \mathbb{E}\left[\int_{D}\left(w_{s}\left(u_{n+1}\right)+f_{n}\right) u_{n+1} d x\right] \\
& +\Delta t\left\|h\left(u_{0}\right)\right\|^{2}+\int_{0}^{t} e^{-c s} \mathbb{E}\left[\left\|h\left(u^{\Delta t}\right)\right\|^{2}\right] d s \\
& -c e^{-c \Delta t} \int_{0}^{(t-\Delta t)^{+}} e^{-c s} \mathbb{E}\left[\left\|u^{\Delta t}\right\|^{2}\right] d s .
\end{aligned}
$$


Note that

$$
\begin{aligned}
\int_{0}^{t} e^{-c s} \mathbb{E}\left[\left\|\nabla u^{\Delta t}\right\|^{2}\right] d s= & \int_{0}^{t} e^{-c s} \mathbb{E}\left[\left\|\nabla\left(u^{\Delta t}-u_{\epsilon}\right)\right\|^{2}+2 \int_{D} \nabla u^{\Delta t} \nabla u_{\epsilon} d x-\left\|\nabla u_{\epsilon}\right\|^{2}\right] d s, \\
\int_{0}^{t} e^{-c s} \mathbb{E}\left[\int_{D} \epsilon\left(u^{\Delta t}\right) u^{\Delta t} d x\right] d s= & \int_{0}^{t} e^{-c s} \mathbb{E}\left[\int_{D}\left(\epsilon\left(u^{\Delta t}\right)-\psi_{\epsilon}\left(u_{\epsilon}\right)\right)\left(u^{\Delta t}-u_{\epsilon}\right) d x\right] d s \\
& +\int_{0}^{t} e^{-c s} \mathbb{E}\left[\int_{D} \epsilon\left(u_{\epsilon}\right)\left(u^{\Delta t}-u_{\epsilon}\right)+\psi_{\epsilon}\left(u^{\Delta t}\right) u_{\epsilon} d x\right] d s,
\end{aligned}
$$

and

$$
\int_{0}^{t} e^{-c s} \mathbb{E}\left[\left\|h\left(u^{\Delta t}\right)\right\|^{2}\right] d s=\int_{0}^{t} e^{-c s} \mathbb{E}\left[\left\|h\left(u^{\Delta t}\right)-h\left(u_{\epsilon}\right)\right\|^{2}+2 \int_{D} h\left(u^{\Delta t}\right) h\left(u_{\epsilon}\right) d x-\left\|h\left(u_{\epsilon}\right)\right\|^{2}\right] d s .
$$

Besides, there exists a constant $C>0$ independent of $\Delta t$ such that

$$
\begin{aligned}
& \left|\sum_{n=0}^{k} e^{-c n \Delta t} \Delta t \mathbb{E}\left[\int_{D} w_{s}\left(u_{n+1}\right) u_{n+1} d x\right]-\int_{0}^{t} e^{-c s} \mathbb{E}\left[\int_{D} w_{s}\left(u^{\Delta t}\right) u^{\Delta t} d x\right] d s\right| \\
\leq & \left|\sum_{n=0}^{k} e^{-c n \Delta t} \Delta t \mathbb{E}\left[\int_{D} w_{s}\left(u_{n+1}\right) u_{n+1} d x\right]-\int_{0}^{t_{k+1}} e^{-c s} \mathbb{E}\left[\int_{D} w_{s}\left(u^{\Delta t}\right) u^{\Delta t} d x\right] d s\right| \\
& +\left|\int_{t}^{t_{k+1}} e^{-c s} \mathbb{E}\left[\int_{D} w_{s}\left(u^{\Delta t}\right) u^{\Delta t} d x\right] d s\right| \\
\leq & \left|\sum_{n=0}^{k} \int_{t_{n}}^{t_{n+1}}\left(e^{-c n \Delta t}-e^{-c s}\right) \mathbb{E}\left[\int_{D} w_{s}\left(u_{n+1}\right) u_{n+1} d x\right] d s\right|+C L_{s} \Delta t \sup _{k} \mathbb{E}\left[\left\|u_{k}\right\|_{L^{2}(D)}^{2}\right] \\
\leqslant & c \sum_{n=0}^{k} \int_{t_{n}}^{t_{n+1}} \Delta t \mathbb{E}\left[\int_{D}\left|w_{s}\left(u_{n+1}\right) u_{n+1}\right| d x\right] d s+C L_{s} \Delta t \\
\leqslant & c \Delta t L_{s}\left\|u^{\Delta t}\right\|_{L^{2}(\Omega \times Q)}^{2}+C L_{s} \Delta t \\
\leqslant & C \Delta t
\end{aligned}
$$

and similarly

$$
\begin{aligned}
& \left|\sum_{n=0}^{k} e^{-c n \Delta t} \Delta t \mathbb{E}\left[\int_{D} f_{n} u_{n+1} d x\right]-\int_{0}^{t} e^{-c s} \mathbb{E}\left[\int_{D} f_{\Delta t} u^{\Delta t} d x\right] d s\right| \\
\leq & \left|\sum_{n=0}^{k} e^{-c n \Delta t} \Delta t \mathbb{E}\left[\int_{D} f_{n} u_{n+1} d x\right]-\int_{0}^{t_{k+1}} e^{-c s} \mathbb{E}\left[\int_{D} f_{\Delta t} u^{\Delta t} d x\right] d s\right| \\
& +\left|\int_{t}^{t_{k+1}} e^{-c s} \mathbb{E}\left[\int_{D} f_{\Delta t} u^{\Delta t} d x\right] d s\right| \\
= & \left|\sum_{n=0}^{k} \int_{t_{n}}^{t_{n+1}}\left(e^{-c n \Delta t}-e^{-c s}\right) \mathbb{E}\left[\int_{D} f_{n} u_{n+1} d x\right] d s\right|+\left|\int_{t}^{t_{k+1}} e^{-c s} \mathbb{E}\left[\int_{D} f_{k} u_{k+1} d x\right] d s\right| \\
\leqslant & c \Delta t|| f_{\Delta t}\left\|_{L^{2}(\Omega \times Q)}\right\| u^{\Delta t}\left\|_{L^{2}(\Omega \times Q)}+\int_{t}^{t_{k+1}}\right\| f_{k}\left\|_{L^{2}(\Omega \times D)}\right\| u_{k+1} \|_{L^{2}(\Omega \times D)} d s \\
\leqslant & c \Delta t|| f_{\Delta t}\left\|_{L^{2}(\Omega \times Q)}\right\| u^{\Delta t}\left\|_{L^{2}(\Omega \times Q)}+\right\| u^{\Delta t} \|_{L^{\infty}\left(0, T ; L^{2}(\Omega \times D)\right)} \sqrt{\Delta t}\left(\int_{t}^{t_{k+1}}\left\|f_{k}\right\|_{L^{2}(\Omega \times D)}^{2} d s\right)^{1 / 2} \\
\leqslant & C \Delta t+\tilde{\epsilon}(\Delta t), \quad \text { where } \tilde{\epsilon}(\Delta t) \rightarrow 0 \text { when } \Delta t \rightarrow 0,
\end{aligned}
$$

as well as

$$
\begin{aligned}
& c e^{-c \Delta t} \int_{0}^{(t-\Delta t)^{+}} e^{-c s} \mathbb{E}\left[\left\|u^{\Delta t}\right\|^{2}\right] d s \\
= & c e^{-c \Delta t} \int_{0}^{t} e^{-c s} \mathbb{E}\left[\left\|u^{\Delta t}\right\|^{2}\right] d s-c e^{-c \Delta t} \int_{(t-\Delta t)^{+}}^{t} e^{-c s} \mathbb{E}\left[\left\|u^{\Delta t}\right\|^{2}\right] d s \\
\geqslant & c e^{-c \Delta t} \int_{0}^{t} e^{-c s} \mathbb{E}\left[\left\|u^{\Delta t}\right\|^{2}\right] d s-c e^{-c \Delta t} \Delta t\left\|u^{\Delta t}\right\|_{L^{\infty}\left(0, T ; L^{2}(\Omega \times D)\right)}^{2} \\
= & c e^{-c \Delta t} \int_{0}^{t} e^{-c s} \mathbb{E}\left[\left\|u^{\Delta t}-u_{\epsilon}\right\|^{2}+2 \int_{D} u^{\Delta t} u_{\epsilon} d x-\left\|u_{\epsilon}\right\|^{2}\right] d s-C \Delta t .
\end{aligned}
$$

12 
Using these, we can write inequality (2.5) in the following way:

$$
\begin{aligned}
& e^{-c t} \mathbb{E}\left[\left\|u^{\Delta t}(t)\right\|^{2}\right]+2 \int_{0}^{t} e^{-c s} \mathbb{E}\left[\left\|\nabla\left(u^{\Delta t}-u_{\epsilon}\right)\right\|^{2}+2 \int_{D} \nabla u^{\Delta t} \nabla u_{\epsilon} d x-\left\|\nabla u_{\epsilon}\right\|^{2}\right] d s \\
& +2 \int_{0}^{t} e^{-c s} \mathbb{E}\left[\int_{D}\left(\epsilon\left(u^{\Delta t}\right)-\psi_{\epsilon}\left(u_{\epsilon}\right)\right)\left(u^{\Delta t}-u_{\epsilon}\right) d x\right] d s \\
& +2 \int_{0}^{t} e^{-c s} \mathbb{E}\left[\int_{D} \epsilon\left(u_{\epsilon}\right)\left(u^{\Delta t}-u_{\epsilon}\right)+\psi_{\epsilon}\left(u^{\Delta t}\right) u_{\epsilon} d x\right] d s \\
& \leqslant\left\|u_{0}\right\|^{2}+2 \int_{0}^{t} e^{-c s} \mathbb{E}\left[\int_{D}\left(w_{s}\left(u^{\Delta t}\right)+f_{\Delta t}\right) u^{\Delta t} d x\right] d s+\Delta t\left\|h\left(u_{0}\right)\right\|^{2} \\
& +\int_{0}^{t} e^{-c s} \mathbb{E}\left[\left\|h\left(u^{\Delta t}\right)-h\left(u_{\epsilon}\right)\right\|^{2}+2 \int_{D} h\left(u^{\Delta t}\right) h\left(u_{\epsilon}\right) d x-\left\|h\left(u_{\epsilon}\right)\right\|^{2}\right] d s \\
& -c e^{-c \Delta t} \int_{0}^{t} e^{-c s} \mathbb{E}\left[\left\|u^{\Delta t}-u_{\epsilon}\right\|^{2}+2 \int_{D} u^{\Delta t} u_{\epsilon} d x-\left\|u_{\epsilon}\right\|^{2}\right] d s+C \Delta t+\tilde{\epsilon}(\Delta t) .
\end{aligned}
$$

And so by noting that

$$
\begin{aligned}
\int_{0}^{t} e^{-c s} \mathbb{E}\left[\int_{D} w_{s}\left(u^{\Delta t}\right) u^{\Delta t} d x\right] d s= & \int_{0}^{t} e^{-c s} \mathbb{E}\left[\int_{D}\left(w_{s}\left(u^{\Delta t}\right)-w_{s}\left(u_{\epsilon}\right)\right)\left(u^{\Delta t}-u_{\epsilon}\right) d x\right] d s \\
& +\int_{0}^{t} e^{-c s} \mathbb{E}\left[\int_{D} w_{s}\left(u_{\epsilon}\right)\left(u^{\Delta t}-u_{\epsilon}\right) d x\right] d s \\
& +\int_{0}^{t} e^{-c s} \mathbb{E}\left[\int_{D} w_{s}\left(u^{\Delta t}\right) u_{\epsilon} d x\right] d s
\end{aligned}
$$

we obtain

$$
\begin{aligned}
& e^{-c t} \mathbb{E}\left[\left\|u^{\Delta t}(t)\right\|^{2}\right]+2 \int_{0}^{t} e^{-c s} \mathbb{E}\left[\left\|\nabla\left(u^{\Delta t}-u_{\epsilon}\right)\right\|^{2}+2 \int_{D} \nabla u^{\Delta t} \nabla u_{\epsilon} d x-\left\|\nabla u_{\epsilon}\right\|^{2}\right] d s \\
& +2 \int_{0}^{t} e^{-c s} \mathbb{E}\left[\int_{D} \epsilon\left(u_{\epsilon}\right)\left(u^{\Delta t}-u_{\epsilon}\right)+\psi_{\epsilon}\left(u^{\Delta t}\right) u_{\epsilon} d x\right] d s \\
& \leqslant \\
& \left\|u_{0}\right\|^{2}+2 \int_{0}^{t} e^{-c s} \mathbb{E}\left[\int_{D} f_{\Delta t} u^{\Delta t} d x\right] d s+2 \int_{0}^{t} e^{-c s} \mathbb{E}\left[\int_{D} w_{s}\left(u^{\Delta t}\right) u_{\epsilon} d x\right] d s \\
& +2 \int_{0}^{t} e^{-c s} \mathbb{E}\left[\int_{D} w_{s}\left(u_{\epsilon}\right)\left(u^{\Delta t}-u_{\epsilon}\right) d x\right] d s+\Delta t\left\|h\left(u_{0}\right)\right\|^{2} \\
& +\int_{0}^{t} e^{-c s} \mathbb{E}\left[2 \int_{D} h\left(u^{\Delta t}\right) h\left(u_{\epsilon}\right) d x-\left\|h\left(u_{\epsilon}\right)\right\|^{2}\right] d s \\
& -c e^{-c \Delta t} \int_{0}^{t} e^{-c s} \mathbb{E}\left[2 \int_{D} u^{\Delta t} u_{\epsilon} d x-\left\|u_{\epsilon}\right\|^{2}\right] d s \\
& +c \Delta t+\left(c_{h}^{2}+2 L_{s}-c e^{-c \Delta t}+\frac{2}{\epsilon}\right)\left\|u^{\Delta t}-u_{\epsilon}\right\|_{L^{2}(\Omega \times Q)}^{2}+\tilde{\epsilon}(\Delta t) .
\end{aligned}
$$

By choosing $c$ such that $C_{h}^{2}+2 L_{s}-c e^{-c \Delta t}+\frac{2}{\epsilon} \leqslant 0$, we finally get

$$
\begin{aligned}
& \int_{0}^{T} e^{-c t} \mathbb{E}\left[\left\|u^{\Delta t}(t)\right\|^{2}\right] d t+2 \int_{0}^{T} \int_{0}^{t} e^{-c s} \mathbb{E}\left[2 \int_{D} \nabla u^{\Delta t} \nabla u_{\epsilon} d x-\left\|\nabla u_{\epsilon}\right\|^{2}\right] d s d t \\
& +2 \int_{0}^{T} \int_{0}^{t} e^{-c s} \mathbb{E}\left[\int_{D} \epsilon\left(u_{\epsilon}\right)\left(u^{\Delta t}-u_{\epsilon}\right)+\psi_{\epsilon}\left(u^{\Delta t}\right) u_{\epsilon} d x\right] d s d t \\
& \leqslant \\
& \leqslant\left\|u_{0}\right\|^{2}+2 \int_{0}^{T} \int_{0}^{t} e^{-c s} \mathbb{E}\left[\int_{D} f_{\Delta t} u^{\Delta t} d x\right] d s d t+2 \int_{0}^{T} \int_{0}^{t} e^{-c s} \mathbb{E}\left[\int_{D} w_{s}\left(u^{\Delta t}\right) u_{\epsilon} d x\right] d s d t \\
& +2 \int_{0}^{T} \int_{0}^{t} e^{-c s} \mathbb{E}\left[\int_{D} w_{s}\left(u_{\epsilon}\right)\left(u^{\Delta t}-u_{\epsilon}\right) d x\right] d s d t+T \Delta t\left\|h\left(u_{0}\right)\right\|^{2} \\
& +\int_{0}^{T} \int_{0}^{t} e^{-c s} \mathbb{E}\left[2 \int_{D} h\left(u^{\Delta t}\right) h\left(u_{\epsilon}\right) d x-\left\|h\left(u_{\epsilon}\right)\right\|^{2}\right] d s d t \\
& -c e^{-c \Delta t} \int_{0}^{T} \int_{0}^{t} e^{-c s} \mathbb{E}\left[2 \int_{D} u^{\Delta t} u_{\epsilon} d x-\left\|u_{\epsilon}\right\|^{2}\right] d s d t+C T \Delta t+\tilde{\epsilon}(\Delta t) .
\end{aligned}
$$


Now, by applying the Itô formula to the process $u_{\epsilon}$ and the function $F(t, v)=e^{-c t}\|v\|^{2}$, we get the following energy equality ([2] Theorem 4.17 p. 105, [21] Theorem 4.2.5 p. 75):

$$
\begin{aligned}
& \int_{0}^{T} e^{-c t} \mathbb{E}\left[\left\|u_{\epsilon}(t)\right\|^{2}\right] d t+c \int_{0}^{T} \int_{0}^{t} e^{-c s} \mathbb{E}\left[\left\|u_{\epsilon}\right\|^{2}\right] d s d t \\
& +2 \int_{0}^{T} \int_{0}^{t} e^{-c s} \mathbb{E}\left[\left\|\nabla u_{\epsilon}\right\|^{2}\right] d s d t+2 \int_{0}^{T} \int_{0}^{t} e^{-c s} \mathbb{E}\left[\int_{D} \chi_{\epsilon} u_{\epsilon} d x\right] d s d t \\
= & T\left\|u_{0}\right\|^{2}+2 \int_{0}^{T} \int_{0}^{t} e^{-c s} \mathbb{E}\left[\int_{D}\left(w_{\epsilon}+f\right) u_{\epsilon} d x\right] d s d t+\int_{0}^{T} \int_{0}^{t} e^{-c s} \mathbb{E}\left[\left\|h_{\epsilon}\right\|^{2}\right] d s d t .
\end{aligned}
$$

By replacing $T\left\|u_{0}\right\|^{2}$ in (2.6) using this last equality, we obtain

$$
\begin{aligned}
& \int_{0}^{T} e^{-c t} \mathbb{E}\left[\left\|u^{\Delta t}(t)\right\|^{2}\right] d t+2 \int_{0}^{T} \int_{0}^{t} e^{-c s} \mathbb{E}\left[2 \int_{D} \nabla u^{\Delta t} \nabla u_{\epsilon} d x-\left\|\nabla u_{\epsilon}\right\|^{2}\right] d s d t \\
& +2 \int_{0}^{T} \int_{0}^{t} e^{-c s} \mathbb{E}\left[\int_{D} \epsilon\left(u_{\epsilon}\right)\left(u^{\Delta t}-u_{\epsilon}\right)+\psi_{\epsilon}\left(u^{\Delta t}\right) u_{\epsilon} d x\right] d s d t \\
& \leqslant \int_{0}^{T} e^{-c t} \mathbb{E}\left[\left\|u_{\epsilon}(t)\right\|^{2}\right] d t+c \int_{0}^{T} \int_{0}^{t} e^{-c s} \mathbb{E}\left[\left\|u_{\epsilon}\right\|^{2}\right] d s d t \\
& +2 \int_{0}^{T} \int_{0}^{t} e^{-c s} \mathbb{E}\left[\left\|\nabla u_{\epsilon}\right\|^{2}\right] d s d t+2 \int_{0}^{T} \int_{0}^{t} e^{-c s} \mathbb{E}\left[\int_{D} \chi_{\epsilon} u_{\epsilon} d x\right] d s d t \\
& -2 \int_{0}^{T} \int_{0}^{t} e^{-c s} \mathbb{E}\left[\int_{D}\left(w_{\epsilon}+f\right) u_{\epsilon} d x\right] d s d t-\int_{0}^{T} \int_{0}^{t} e^{-c s} \mathbb{E}\left[\int_{D} h_{\epsilon}^{2} d x\right] d s d t \\
& +2 \int_{0}^{T} \int_{0}^{t} e^{-c s} \mathbb{E}\left[\int_{D} f_{\Delta t} u^{\Delta t} d x\right] d s d t+2 \int_{0}^{t} \int_{0}^{t} e^{-c s} \mathbb{E}\left[\int_{D} w_{s}\left(u^{\Delta t}\right) u_{\epsilon} d x\right] d s d t \\
& +2 \int_{0}^{T} \int_{0}^{t} e^{-c s} \mathbb{E}\left[\int_{D} w_{s}\left(u_{\epsilon}\right)\left(u^{\Delta t}-u_{\epsilon}\right) d x\right] d s d t+T \Delta t\left\|h\left(u_{0}\right)\right\|^{2} \\
& +\int_{0}^{T} \int_{0}^{t} e^{-c s} \mathbb{E}\left[2 \int_{D} h\left(u^{\Delta t}\right) h\left(u_{\epsilon}\right) d x-\left\|h\left(u_{\epsilon}\right)\right\|^{2}\right] d s d t \\
& -c e^{-c \Delta t} \int_{0}^{T} \int_{0}^{t} e^{-c s} \mathbb{E}\left[2 \int u_{D}^{\Delta t} u_{\epsilon} d x-\left\|u_{\epsilon}\right\|^{2}\right] d s d t+C T \Delta t+\tilde{\epsilon}(\Delta t) .
\end{aligned}
$$

And by passing to the superior limit, we get $f_{\Delta t} \underset{\Delta t}{\longrightarrow} f$ in $L^{2}(\Omega \times Q)$ :

$$
\begin{aligned}
& \limsup \int_{\Delta t}^{T} e^{-c t} \mathbb{E}\left[\left\|u^{\Delta t}(t)\right\|^{2}\right] d t+2 \int_{0}^{T} \int_{0}^{t} e^{-c s} \mathbb{E}\left[\left\|\nabla u_{\epsilon}\right\|^{2}\right] d s d t \\
& +2 \int_{0}^{T} \int_{0}^{t} e^{-c s} \mathbb{E}\left[\int_{D} \chi_{\epsilon} u_{\epsilon} d x\right] d s d t \\
& \leqslant \int_{0}^{T} e^{-c t} \mathbb{E}\left[\left\|u_{\epsilon}(t)\right\|^{2}\right] d t+c \int_{0}^{T} \int_{0}^{t} e^{-c s} \mathbb{E}\left[\left\|u_{\epsilon}\right\|^{2}\right] d s d t \\
& +2 \int_{0}^{T} \int_{0}^{t} e^{-c s} \mathbb{E}\left[\left\|\nabla u_{\epsilon}\right\|^{2}\right] d s d t+2 \int_{0}^{T} \int_{0}^{t} e^{-c s} \mathbb{E}\left[\int_{D} \chi_{\epsilon} u_{\epsilon} d x\right] d s d t \\
& -2 \int_{0}^{T} \int_{0}^{t} e^{-c s} \mathbb{E}\left[\int_{D}\left(w_{\epsilon}+f\right) u_{\epsilon} d x\right] d s d t-\int_{0}^{T} \int_{0}^{t} e^{-c s} \mathbb{E}\left[\left\|h_{\epsilon}\right\|^{2}\right] d s d t \\
& +2 \int_{0}^{T} \int_{0}^{t} e^{-c s} \mathbb{E}\left[\int_{D} f u_{\epsilon} d x\right] d s d t+2 \int_{0}^{T} \int_{0}^{t} e^{-c s} \mathbb{E}\left[\int_{D} w_{\epsilon} u_{\epsilon} d x\right] d s d t \\
& +\int_{0}^{T} \int_{0}^{t} e^{-c s} \mathbb{E}\left[2 \int_{D} h_{\epsilon} h\left(u_{\epsilon}\right) d x-\left\|h\left(u_{\epsilon}\right)\right\|^{2}\right] d s d t-c \int_{0}^{T} \int_{0}^{t} e^{-c s} \mathbb{E}\left[\left\|u_{\epsilon}\right\|^{2}\right] d s d t
\end{aligned}
$$

Finally,

$$
\underset{\Delta t}{\limsup } \int_{0}^{T} e^{-c t} \mathbb{E}\left[\left\|u^{\Delta t}(t)\right\|^{2}\right] d t \leqslant \int_{0}^{T} e^{-c t} \mathbb{E}\left[\left\|u_{\epsilon}(t)\right\|^{2}\right] d t-\int_{0}^{T} \int_{0}^{t} e^{-c s} \mathbb{E}\left[\left\|h\left(u_{\epsilon}\right)-h_{\epsilon}\right\|^{2}\right] d s d t .
$$

Thus, $u^{\Delta t} \rightarrow u_{\epsilon}$ in $L^{2}(\Omega \times Q), h\left(u_{\epsilon}\right)=h_{\epsilon}, \chi_{\epsilon}=\psi_{\epsilon}\left(u_{\epsilon}\right)$ and $w_{\epsilon}=w_{s}\left(u_{\epsilon}\right)$. 


\subsection{Uniqueness of $u_{\epsilon}$}

Proposition 2.6

There exists a unique solution to Problem (2.1).

Proof

Let $u_{\epsilon}, \tilde{u}_{\epsilon}$ be two solutions of Problem (2.1) associated with the same initial condition $u_{0}$. Then, $u_{\epsilon}-\tilde{u}_{\epsilon}$ satisfies

$$
d\left(u_{\epsilon}-\tilde{u}_{\epsilon}\right)+\left[\psi_{\epsilon}\left(u_{\epsilon}\right)-\psi_{\epsilon}\left(\tilde{u}_{\epsilon}\right)-\Delta\left(u_{\epsilon}-\tilde{u}_{\epsilon}\right)\right] d t=\left[w_{s}\left(u_{\epsilon}\right)-w_{s}\left(\tilde{u}_{\epsilon}\right)\right] d t+\left[h\left(u_{\epsilon}\right)-h\left(\tilde{u}_{\epsilon}\right)\right] d W .
$$

Let $c$ be a positive constant; by applying the Itô formula $[2,21]$ to the process $u_{\epsilon}-\tilde{u}_{\epsilon}$ and the function $F(t, v)=e^{-c t}\|v\|^{2}$, one gets for any $t \in[0, T]$ :

$$
\begin{aligned}
& e^{-c t} \mathbb{E}\left[\left\|\left(u_{\epsilon}-\tilde{u}_{\epsilon}\right)(t)\right\|^{2}\right]+c \int_{0}^{t} e^{-c s}\left\|u_{\epsilon}-\tilde{u}_{\epsilon}\right\|^{2} d s+2 \int_{0}^{t} e^{-c s} \mathbb{E}\left[\left\|\nabla\left(u_{\epsilon}-\tilde{u}_{\epsilon}\right)\right\|^{2}\right] d s \\
& +2 \int_{0}^{t} e^{-c s} \mathbb{E}\left[\left(\psi_{\epsilon}\left(u_{\epsilon}\right)-\psi_{\epsilon}\left(\tilde{u}_{\epsilon}\right)\right)\left(u_{\epsilon}-\tilde{u}_{\epsilon}\right)\right] d s \\
= & 2 \int_{0}^{t} e^{-c s} \mathbb{E}\left[\left(w_{s}\left(u_{\epsilon}\right)-w_{s}\left(\tilde{u}_{\epsilon}\right)\right)\left(u_{\epsilon}-\tilde{u}_{\epsilon}\right)\right] d s+\int_{0}^{t} e^{-c s} \mathbb{E}\left[\left\|h\left(u_{\epsilon}\right)-h\left(\tilde{u}_{\epsilon}\right)\right\|^{2}\right] d s .
\end{aligned}
$$

In this way, by choosing $c$ such that $2 L_{s}+C_{h}^{2}-c<0$, one gets

$$
\forall t \in l, \quad e^{-c t} \mathbb{E}\left[\left\|\left(u_{\epsilon}-\tilde{u}_{\epsilon}\right)(t)\right\|^{2}\right] \leqslant 0,
$$

which proves the announced uniqueness result.

\section{Proof of the main result}

The aim of this section is to prove our main result, Theorem 1.2. To do this, firstly, we show the following convergence result.

Proposition 3.1

Up to subsequences, $\left(u_{\epsilon}\right)_{\epsilon>0}$ converges strongly in $\mathcal{N}_{w}^{2}\left(0, T, H^{1}(D)\right)$ and weakly* in $L^{\infty}\left(0, T ; L^{2}(\Omega \times D)\right)$ to a function $u_{,}\left(\psi_{\epsilon}\left(u_{\epsilon}\right)\right)_{\epsilon>0}$ converges weakly in $\mathcal{N}_{w}^{2}\left(0, T, L^{2}(D)\right)$ to a function $\xi \in \partial I_{[0,1]}(u)$, and in addition, almost everywhere in $(0, T), \mathbb{P}$-almost surely in $\Omega$, and for any $v$ in $H^{1}(D)$

$$
<\partial_{t}\left(u-\int_{0} h(u) d W\right), v>_{v^{\prime}, v}+\int_{D} \nabla u . \nabla v d x+\int_{D} \xi v d x=\int_{D}\left(w_{s}(u)+f\right) v d x
$$

3.1. Study of the sequences $\left(u_{\epsilon}\right)_{\epsilon>0}$ and $\left(\psi_{\epsilon}\left(u_{\epsilon}\right)\right)_{\epsilon>0}$

Proposition 3.2

$\left(u_{\epsilon}\right)_{\epsilon>0}$ is bounded in $L^{\infty}\left(0, T ; L^{2}(\Omega \times D)\right) \cap \mathcal{N}_{w}^{2}\left(0, T, H^{1}(D)\right)$.

Proof

This first result is a direct consequence of Proposition 2.2 and of the lower semi-continuity of the norm for the weak, or weak-*, convergence.

Proposition 3.3

$\left(\psi_{\epsilon}\left(u_{\epsilon}\right)\right)_{\epsilon>0}$ is bounded in $\mathcal{N}_{w}^{2}\left(0, T, L^{2}(D)\right)$.

Proof

We consider the convex antiderivative $\phi_{\epsilon}$ of $\psi_{\epsilon}$ defined by the following:

$$
\phi_{\epsilon}(v)=\left\{\begin{array}{cl}
\frac{v^{2}}{2 \epsilon} & \text { if } v \leqslant 0 \\
0 & \text { if } v \in[0,1] \\
\frac{(v-1)^{2}}{2 \epsilon} & \text { if } v \geqslant 1
\end{array}\right.
$$


By applying the Itô formula $[2,21]$ to the process $u_{\epsilon}$ and the function $F(t, v)=\int_{D} \phi_{\epsilon}(v) d x$, one gets for any $t \in[0, T]$ :

$$
\begin{aligned}
\int_{D} \phi_{\epsilon}\left(u_{\epsilon}(t)\right) d x= & \int_{D} \phi_{\epsilon}\left(u_{0}\right) d x+\int_{0}^{t} \int_{D} \psi_{\epsilon}\left(u_{\epsilon}\right)\left\{\Delta u_{\epsilon}-\psi_{\epsilon}\left(u_{\epsilon}\right)+w_{s}\left(u_{\epsilon}\right)+f\right\} d x d s \\
& +\int_{0}^{t} \int_{D} \psi_{\epsilon}\left(u_{\epsilon}\right) h\left(u_{\epsilon}\right) d x d W(s)+\frac{1}{2} \int_{0}^{t} \int_{D}{ }_{\epsilon}^{\prime}\left(u_{\epsilon}\right) h^{2}\left(u_{\epsilon}\right) d x d s .
\end{aligned}
$$

Because $h(0)=h(1)=0$, one gets

$$
\begin{aligned}
& \int_{0}^{t} \int_{D}{ }_{\epsilon}^{\prime}\left(u_{\epsilon}\right) h^{2}\left(u_{\epsilon}\right) d x d s \\
= & \int_{0}^{t} \int_{u_{\epsilon}<0}{ }_{\epsilon}^{\prime}\left(u_{\epsilon}\right) h^{2}\left(u_{\epsilon}\right) d x d s+\int_{0}^{t} \int_{u_{\epsilon}>1}{ }_{\epsilon}^{\prime}\left(u_{\epsilon}\right) h^{2}\left(u_{\epsilon}\right) d x d s \\
= & \int_{0}^{t} \int_{u_{\epsilon}<0}{ }_{\epsilon}^{\prime}\left(u_{\epsilon}\right)\left[h\left(u_{\epsilon}\right)-h(0)\right]^{2} d x d s+\int_{0}^{t} \int_{u_{\epsilon}>1}{ }_{\epsilon}^{\prime}\left(u_{\epsilon}\right)\left[h\left(u_{\epsilon}\right)-h(1)\right]^{2} d x d s \\
\leqslant & C_{h}^{2} \int_{0}^{t} \int_{u_{\epsilon}<0}{ }_{\epsilon}^{\prime}\left(u_{\epsilon}\right) u_{\epsilon}^{2} d x d s+C_{h}^{2} \int_{0}^{t} \int_{u_{\epsilon}>1}{ }_{\epsilon}^{\prime}\left(u_{\epsilon}\right)\left(u_{\epsilon}-1\right)^{2} d x d s \\
\leqslant & 2 C_{h}^{2} \int_{0}^{t} \int_{D} \phi_{\epsilon}\left(u_{\epsilon}(s)\right) d x d s .
\end{aligned}
$$

After taking the expectation in (3.1), one gets $\phi_{\epsilon}\left(u_{0}\right)=0$ :

$$
\begin{aligned}
& \mathbb{E}\left[\int_{D} \phi_{\epsilon}\left(u_{\epsilon}(t)\right) d x\right]+\mathbb{E}\left[\int_{0}^{t} \int_{D} \psi_{\epsilon}^{\prime}\left(u_{\epsilon}\right)\left|\nabla u_{\epsilon}\right|^{2} d x d s\right]+\left\|\psi_{\epsilon}\left(u_{\epsilon}\right)\right\|_{L^{2}(\Omega \times Q)}^{2} \\
\leqslant & \frac{1}{2}\left\|\epsilon_{\epsilon}\left(u_{\epsilon}\right)\right\|_{L^{2}(\Omega \times Q)}^{2}+\left\|w_{s}\left(u_{\epsilon}\right)\right\|_{L^{2}(\Omega \times Q)}^{2}+\|f\|_{L^{2}(\Omega \times Q)}^{2}+C_{h}^{2} \mathbb{E}\left[\int_{0}^{t} \int_{D} \phi_{\epsilon}\left(u_{\epsilon}(s)\right) d x d s\right],
\end{aligned}
$$

and using the fact that $\psi_{\epsilon}^{\prime} \geqslant 0$, one gets

$$
\begin{aligned}
& \mathbb{E}\left[\int_{D} \phi_{\epsilon}\left(u_{\epsilon}(t)\right) d x\right]+\frac{1}{2}\left\|\epsilon_{\epsilon}\left(u_{\epsilon}\right)\right\|_{L^{2}(\Omega \times Q)}^{2} \\
\leqslant & \left\|w_{s}\left(u_{\epsilon}\right)\right\|_{L^{2}(\Omega \times Q)}^{2}+\|f\|_{L^{2}(\Omega \times Q)}^{2}+2 C_{h}^{2} \mathbb{E}\left[\int_{0}^{t} \int_{D} \phi_{\epsilon}\left(u_{\epsilon}(s)\right) d x d s\right] .
\end{aligned}
$$

Because $\phi_{\epsilon}\left(u_{\epsilon}(t)\right) \geqslant 0$, by using Gronwall's lemma, one gets the existence of $\tilde{C}>0$ independent of $\epsilon$ such that

$$
2 C_{h}^{2} \mathbb{E}\left[\int_{0}^{t} \int_{D} \phi_{\epsilon}\left(u_{\epsilon}(s)\right) d x d s\right] \leqslant \tilde{C}
$$

thus,

$$
\left\|\psi_{\epsilon}\left(u_{\epsilon}\right)\right\|_{L^{2}(\Omega \times Q)}^{2} \leqslant 2 L_{s}^{2}\left\|u_{\epsilon}\right\|_{L^{2}(\Omega \times Q)}^{2}+2\|f\|_{L^{2}(\Omega \times Q)}^{2}+2 C,
$$

and then $\left(\psi_{\epsilon}\left(u_{\epsilon}\right)\right)_{\epsilon>0}$ is bounded in $\mathcal{N}_{w}^{2}\left(0, T, L^{2}(D)\right)$ thanks to Proposition 3.2.

Using this result, the convergence of $u^{\Delta t}$ to $u_{\epsilon}$ in $L^{2}(\Omega \times Q)$ and Proposition 2.3, one gets the following bounds, in particular for the sequences $\left(\partial_{t}\left(u_{\epsilon}-\int_{0} h\left(u_{\epsilon}\right) d W\right)\right)_{\epsilon>0^{\prime}}\left(\frac{u_{\epsilon}^{-}}{\epsilon}\right)_{\epsilon>0}$ and $\left(\frac{\left(u_{\epsilon}-1\right)^{+}}{\epsilon}\right)_{\epsilon>0}$.

Proposition 3.4

There exists a constant $C>0$ that only depends on $T, C_{h}, u_{0}, L_{s}$, and $f$ such that

$$
\left\|u_{\epsilon}\right\|_{L^{\infty}\left(0, T_{i} L^{2}\left(\Omega ; H^{1}(D)\right)\right)},\left\|\partial_{t}\left(u_{\epsilon}-\int_{0}^{.} h\left(u_{\epsilon}\right) d W\right)\right\|_{L^{2}(\Omega \times Q)},\left\|\Delta u_{\epsilon}\right\|_{L^{2}(\Omega \times Q)} \leqslant C .
$$

Proof

The boundedness of $\left\|u_{\epsilon}\right\|_{L^{\infty}\left(0, T ; L^{2}\left(\Omega ; H^{1}(D)\right)\right)}$ is a direct consequence of Proposition 2.3 and the lower semi-continuity of the norm for the weak-* convergence. Moreover, thanks to Proposition 2.3, there exists a constant $C>0$ that only depends on $T, C_{h}, u_{0}, L_{s}$, and $f$ such that

$$
\left\|\partial_{t}\left(\tilde{u}^{\Delta t}-\tilde{B}^{\Delta t}\right)\right\|_{L^{2}(\Omega \times Q)} \leqslant C\left(1+\left\|\psi_{\epsilon}\left(u^{\Delta t}\right)\right\|_{L^{2}(\Omega \times Q)}\right) .
$$


Then, using the lower semi-continuity of the norm for the weak convergence, the strong convergence of $\psi_{\epsilon}\left(u^{\Delta t}\right)$ towards $\psi_{\epsilon}\left(u_{\epsilon}\right)$ in $L^{2}(\Omega \times Q)$ given by Propositions 2.5 and 3.3, there exists $\tilde{C}$ that only depends on $T, C_{h}, u_{0}, L_{s}$, and $f$ such that

$$
\begin{aligned}
\left\|\partial_{t}\left(u_{\epsilon}-\int_{0} h\left(u_{\epsilon}\right) d W\right)\right\|_{L^{2}(\Omega \times Q)} & \leqslant \liminf _{\Delta t}\left\|\partial_{t}\left(\tilde{u}^{\Delta t}-\tilde{B}^{\Delta t}\right)\right\|_{L^{2}(\Omega \times Q)} \\
& \leqslant C\left(1+\liminf _{\Delta t}\left\{\left\|\psi_{\epsilon}\left(u^{\Delta t}\right)-\epsilon_{\epsilon}\left(u_{\epsilon}\right)\right\|_{L^{2}(\Omega \times Q)}+\left\|\psi_{\epsilon}\left(u_{\epsilon}\right)\right\|_{L^{2}(\Omega \times Q)}\right\}\right) \\
& \leqslant \tilde{C} .
\end{aligned}
$$

Finally, a comparison in (2.1a) gives the boundedness of $\Delta u_{\epsilon}$ in $L^{2}(\Omega \times Q)$.

Proposition 3.5

$\left(\frac{u_{\epsilon}^{-}}{\epsilon}\right)_{\epsilon>0}$ and $\left(\frac{\left(u_{\epsilon}-1\right)^{+}}{\epsilon}\right)_{\epsilon>0}$ are bounded in $L^{2}(\Omega \times Q)$.

Proof

Because $\psi_{\epsilon}\left(u_{\epsilon}\right)=-\frac{u_{\epsilon}^{-}}{\epsilon}+\frac{\left(u_{\epsilon}-1\right)^{+}}{\epsilon}$ is bounded in $L^{2}(\Omega \times Q)$ and $u_{\epsilon}^{-}\left(u_{\epsilon}-1\right)^{+}=0$, one gets that

$$
\left\|-\frac{u_{\epsilon}^{-}}{\epsilon}\right\|_{L^{2}(\Omega \times Q)}^{2}+\left\|\frac{\left(u_{\epsilon}-1\right)^{+}}{\epsilon}\right\|_{L^{2}(\Omega \times Q)}^{2}=\left\|-\frac{u_{\epsilon}^{-}}{\epsilon}+\frac{\left(u_{\epsilon}-1\right)^{+}}{\epsilon}\right\|_{L^{2}(\Omega \times Q)}^{2}=\left\|\psi_{\epsilon}\left(u_{\epsilon}\right)\right\|_{L^{2}(\Omega \times Q)}^{2} \leqslant C,
$$

and the result holds.

\subsection{Existence of a solution}

Proposition 3.6

There exist $u \in \mathcal{N}_{w}^{2}\left(0, T, H^{1}(D)\right) \cap L^{\infty}\left(0, T, L^{2}\left(\Omega, H^{1}(D)\right)\right) \cap L^{2}\left(\Omega, \mathscr{C}\left(0, T ; L^{2}(D)\right)\right), \xi, \xi_{1}, \xi_{2}$ in $\mathcal{N}_{w}^{2}\left(0, T, L^{2}(D)\right)$ and $\bar{w}_{s}, \bar{h}$ in $\mathcal{N}_{w}^{2}\left(0, T, H^{1}(D)\right)$ such that up to subsequences denoted in the same way, the following convergences hold as $\epsilon \rightarrow 0$ :

$$
\begin{aligned}
u_{\epsilon} & \rightarrow u \text { in } \mathcal{N}_{w}^{2}\left(0, T ; H^{1}(D)\right), \\
u_{\epsilon} & \stackrel{*}{\rightarrow} u \text { in } L^{\infty}\left(0, T, L^{2}\left(\Omega, H^{1}(D)\right)\right), \\
h\left(u_{\epsilon}\right) & \rightarrow \bar{h} \text { in } L^{2}\left(\Omega \times(0, T), H^{1}(D)\right), \\
u_{\epsilon}-\int_{0} h\left(u_{\epsilon}\right) d W & \rightarrow u-\int_{0}^{\cdot} \bar{h} d W \text { in } L^{2}\left(\Omega, H^{1}(Q)\right), \\
w_{s}\left(u_{\epsilon}\right) & \rightarrow \bar{w}_{s} \text { in } L^{2}\left(\Omega \times(0, T), H^{1}(D)\right), \\
\epsilon\left(u_{\epsilon}\right) & \rightarrow \xi \text { in } L^{2}(\Omega \times Q), \\
-\frac{u_{\epsilon}^{-}}{\epsilon} & \rightarrow \xi_{1} \text { in } L^{2}(\Omega \times Q), \text { with } \xi_{1} \leqslant 0, \\
\frac{\left(u_{\epsilon}-1\right)^{+}}{\epsilon} & \rightarrow \xi_{2} \text { in } L^{2}(\Omega \times Q), \text { with } \xi_{2} \geqslant 0 .
\end{aligned}
$$

Proof

These results are immediate using estimates given by Propositions 3.2, 3.3, 3.4, and 3.5. Let us precise briefly that the regularity $u \in$ $L^{2}\left(\Omega, \mathscr{C}\left(0, T ; L^{2}(D)\right)\right)$ comes from the fact that

$$
u-\int_{0}^{\cdot} \bar{h} d W \in L^{2}\left(\Omega, H^{1}(Q)\right) \hookrightarrow L^{2}\left(\Omega, \mathscr{C}\left(0, T ; L^{2}(D)\right)\right),
$$

and the result holds because $\int_{0} \bar{h} d W \in L^{2}\left(\Omega, \mathscr{C}\left(0, T ; L^{2}(D)\right)\right)$ ([2] Theorem 4.12 p. 101).

Note also that $\xi_{1} \leq 0$ (resp. $\xi_{2} \geq 0$ ) because $-\frac{u_{\epsilon}^{-}}{\epsilon} \leq 0$ (resp. ${\frac{\left(u_{\epsilon}-1\right)}{\epsilon}}^{+} \geq 0$ ) and as the subset of the non-negative elements of $L^{2}(\Omega \times Q)$ is a closed convex set.

Remark 3.7

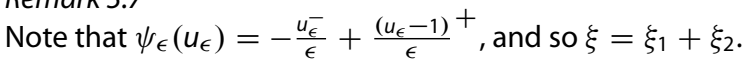

Proposition 3.8

Up to a subsequence $u_{\epsilon}(T) \rightarrow u(T)$ in $L^{2}(\Omega \times D)$.

Proof

Because $u_{\epsilon}-\int_{0} h\left(u_{\epsilon}\right) d W$ converges weakly to $u-\int_{0} \bar{h} d W$ in $L^{2}\left(\Omega, H^{1}(Q)\right)$, it converges weakly in

$$
L^{2}\left(\Omega, \mathscr{C}\left(0, T ; L^{2}(D)\right)\right) \hookrightarrow \mathscr{C}\left(0, T ; L^{2}(\Omega \times D)\right) .
$$

Moreover, using the linear continuity of the stochastic integral, we have

$$
\int_{0}^{T} h\left(u_{\epsilon}\right) d W \rightarrow \int_{0}^{T} \bar{h} d W \text { in } L^{2}(\Omega \times D) .
$$


We deduce that $u_{\epsilon}(T) \rightarrow u(T)$ in $L^{2}(\Omega \times D)$.

Remark 4

In the same manner, because $u_{\epsilon}(., 0)=u_{0}$, one shows that $u(., 0)=u_{0}$ in the sense that

$$
\mathbb{P} \text {-a.s in } \Omega, u(., 0)=\lim _{t \rightarrow 0} u(., t) \text { in } L^{2}(D) \text {. }
$$

Proposition 3.9

$u_{\epsilon}^{-} \rightarrow 0$ and $\left(u_{\epsilon}-1\right)^{+} \rightarrow 0$ in $L^{2}(\Omega \times Q)$ as $\epsilon \rightarrow 0$.

Proof

This result is a direct consequence of Proposition 3.5. Because there exists $C>0$ such that

$$
\left\|-\frac{u_{\epsilon}^{-}}{\epsilon}\right\|_{L^{2}(\Omega \times Q)}^{2}+\left\|\frac{\left(u_{\epsilon}-1\right)^{+}}{\epsilon}\right\|_{L^{2}(\Omega \times Q)}^{2} \leqslant C
$$

one gets

$$
\left\|u_{\epsilon}^{-}\right\|_{L^{2}(\Omega \times Q)}^{2}+\left\|\left(u_{\epsilon}-1\right)^{+}\right\|_{L^{2}(\Omega \times Q)}^{2} \leqslant C \epsilon^{2}
$$

and the result holds.

Remark 3.10

Because

$$
\psi_{\epsilon}\left(u_{\epsilon}\right) u_{\epsilon}=\frac{u_{\epsilon}^{-}}{\epsilon} \times u_{\epsilon}^{-}+\frac{\left(u_{\epsilon}-1\right)^{+}}{\epsilon} \times\left(\left(u_{\epsilon}-1\right)^{+}+1\right),
$$

one gets that

$$
\mathbb{E}\left[\int_{Q} \psi_{\epsilon}\left(u_{\epsilon}\right) u_{\epsilon} d x d s\right] \rightarrow \mathbb{E}\left[\int_{Q} \xi_{2} d x d s\right] \text { as } \epsilon \rightarrow 0
$$

By passing to the limit in the variational formulation (2.3), one gets that for any $v$ in $H^{1}(D), \alpha$ in $L^{2}(0, T)$, and any $\beta$ in $L^{2}(\Omega)$,

$$
\begin{aligned}
& \int_{\Omega} \int_{0}^{T} \int_{D} \partial_{t}\left(u-\int_{0}^{\cdot} \bar{h}(s) d W(s)\right) v d x \alpha \beta d t d \mathbb{P}+\int_{\Omega \times Q}(\nabla u . \nabla v) \alpha \beta d x d t d \mathbb{P} \\
+ & \int_{\Omega \times Q} \xi v \alpha \beta d x d t d \mathbb{P}=\int_{\Omega \times Q}\left(\bar{w}_{s}+f\right) v \alpha \beta d x d t d \mathbb{P} .
\end{aligned}
$$

Because $H^{1}(D)$ is separable, one gets that almost everywhere in $(0, T), \mathbb{P}$-almost surely in $\Omega$ and for any $v$ in $H^{1}(D)$ :

$$
\int_{D} \partial_{t}\left(u-\int_{0}^{\cdot} \bar{h}(s) d W(s)\right) v d x+\int_{D} \nabla u . \nabla v d x+\int_{D} \xi v d x=\int_{D}\left(\bar{w}_{s}+f\right) v d x
$$

In particular, $u$ is a continuous $L^{2}(D)$-valued predictable process satisfying

$$
u(t)=u(0)+\int_{0}^{t}\left(\Delta u-\xi+\bar{w}_{s}+f\right) d s+\int_{0}^{t} \bar{h}(s) d W(s)
$$

where $\Delta$ denotes the Laplace operator on $H^{1}(D)$ associated with the formal Neumann boundary condition. Note that in our situation, $\Delta u$ is an element of $L^{2}(D), \nabla u$ is then an element of $H(\operatorname{div}, D)$, and the normal trace $\nabla u . n$ exists in $H^{-1 / 2}(\partial D)$. Moreover, the solution $u$ satisfies the following energy equality $\mathbb{P}$-almost surely in $\Omega$ and for any $t \in[0, T]$ by denoting as previously $\|\|=.\|.\|_{L^{2}(D)}$ and because $u(0)=u_{0}$

Proposition 3.10

$$
\mathbb{E}\left[\|u(t)\|^{2}\right]=\left\|u_{0}\right\|^{2}-2 \mathbb{E}\left[\int_{0}^{t}\|\nabla u\|^{2} d s\right]+2 \mathbb{E}\left[\int_{0}^{t} \int_{D} u\left(f-\xi+\bar{w}_{s}\right) d x d s\right]+\mathbb{E}\left[\int_{0}^{t}\|\bar{h}\|^{2} d s\right] .
$$

$\xi \in \partial I_{[0,1]}(u), \bar{h}=h(u), \bar{w}_{s}=w_{s}(u)$, and the convergence of $u_{\epsilon}$ towards $u$ holds strongly in $L^{2}\left((0, T) \times \Omega, H^{1}(D)\right)$.

Proof

We use here the same type of arguments as in the proof of Proposition 2.5. By applying the Itô formula to the process $u_{\epsilon}$ and the function $F(t, v)=\frac{1}{2} e^{-\alpha t}\|v\|^{2}$ with $\alpha>0$, one gets that

$$
\begin{gathered}
e^{-\alpha T} \frac{1}{2}\left\|u_{\epsilon}(T)\right\|^{2}-\frac{1}{2}\left\|u_{0}\right\|^{2}+\frac{1}{2} \int_{Q} \alpha e^{-\alpha s}\left|u_{\epsilon}\right|^{2} d x d s+\int_{Q} e^{-\alpha s}\left|\nabla u_{\epsilon}\right|^{2} d x d s+\int_{Q} e^{-\alpha s} u_{\epsilon} \psi_{\epsilon}\left(u_{\epsilon}\right) d x d s \\
=\int_{Q} e^{-\alpha s} u_{\epsilon}\left(w_{s}\left(u_{\epsilon}\right)+f\right) d x d s+\int_{0}^{T} \int_{D} e^{-\alpha s} u_{\epsilon} h\left(u_{\epsilon}\right) d x d W(s)+\frac{1}{2} \int_{Q} e^{-\alpha s} h^{2}\left(u_{\epsilon}\right) d x d s .
\end{gathered}
$$


Thus, by taking the expectation,

$$
\begin{aligned}
& e^{-\alpha T} \frac{1}{2} \mathbb{E}\left[\left\|u_{\epsilon}(T)\right\|^{2}\right]-\frac{1}{2}\left\|u_{0}\right\|^{2}+\mathbb{E}\left[\int_{Q} e^{-\alpha s}\left|\nabla u_{\epsilon}\right|^{2} d x d s\right]+\mathbb{E}\left[\int_{Q} e^{-\alpha s} u_{\epsilon} \psi_{\epsilon}\left(u_{\epsilon}\right) d x d s\right] \\
& +\mathbb{E}\left[\int_{Q} e^{-\alpha s}\left(\frac{\alpha}{2}\left|u_{\epsilon}\right|^{2}-u_{\epsilon} w_{s}\left(u_{\epsilon}\right)-f u_{\epsilon}-\frac{1}{2} h^{2}\left(u_{\epsilon}\right)\right) d x d s\right]=0 .
\end{aligned}
$$

Now, by passing to the superior limit, we get using Remark 3.10

$$
\begin{aligned}
& \limsup \left(e^{-\alpha T} \frac{1}{2} \mathbb{E}\left[\left\|u_{\epsilon}(T)\right\|^{2}\right]+\mathbb{E}\left[\int_{Q} e^{-\alpha s}\left|\nabla u_{\epsilon}\right|^{2} d x d s\right]\right)+\mathbb{E}\left[\int_{Q} e^{-\alpha s} \xi_{2} d x d s\right] \\
& +\liminf \left(\mathbb{E}\left[\int_{Q} e^{-\alpha s}\left(\frac{\alpha}{2}\left|u_{\epsilon}\right|^{2}-u_{\epsilon} w_{s}\left(u_{\epsilon}\right)-\frac{1}{2} h^{2}\left(u_{\epsilon}\right)\right) d x d s\right]\right) \\
& \leqslant \mathbb{E}\left[\int_{Q} e^{-\alpha s} f u d x d s\right]+\frac{1}{2}\left\|u_{0}\right\|^{2} .
\end{aligned}
$$

By denoting

$$
A_{\epsilon}=\mathbb{E}\left[\int_{Q} e^{-\alpha s}\left(\frac{\alpha}{2}\left|u_{\epsilon}\right|^{2}-u_{\epsilon} w_{s}\left(u_{\epsilon}\right)-\frac{1}{2} h^{2}\left(u_{\epsilon}\right)\right) d x d s\right],
$$

we have

$$
\begin{aligned}
A_{\epsilon}= & \mathbb{E}\left[\int_{Q} e^{-\alpha s}\left\{\frac{\alpha}{2}\left|u_{\epsilon}-u\right|^{2}-\left(u_{\epsilon}-u\right)\left(w_{s}\left(u_{\epsilon}\right)-w_{s}(u)\right)-\frac{1}{2}\left(h\left(u_{\epsilon}\right)-h(u)\right)^{2}\right\} d x d s\right] \\
& +\mathbb{E}\left[\int_{Q} e^{-\alpha s}\left\{\alpha\left(u_{\epsilon} u-\frac{u^{2}}{2}\right)-u_{\epsilon} w_{s}(u)-u\left(w_{s}\left(u_{\epsilon}\right)-w_{s}(u)\right)-h(u) h\left(u_{\epsilon}\right)+\frac{1}{2} h^{2}(u)\right\} d x d s\right] \\
\geqslant & \mathbb{E}\left[\int_{Q} e^{-\alpha s}\left|u_{\epsilon}-u\right|^{2}\left(\frac{\alpha}{2}-L_{s}-\frac{C_{h}^{2}}{2}\right) d x d s\right]+\mathbb{E}\left[\int_{Q} \frac{\alpha}{2} e^{-\alpha s}\left(u_{\epsilon} u+u\left(u_{\epsilon}-u\right)\right) d x d s\right] \\
+ & \mathbb{E}\left[\int_{Q} e^{-\alpha s}\left\{\left(u-u_{\epsilon}\right) w_{s}(u)-u w_{s}\left(u_{\epsilon}\right)-h(u) h\left(u_{\epsilon}\right)+\frac{1}{2} h^{2}(u)\right\} d x d s\right] .
\end{aligned}
$$

Thus,

$$
\begin{aligned}
\liminf A_{\epsilon} \geqslant & \liminf \mathbb{E}\left[\int_{Q} e^{-\alpha s}\left|u_{\epsilon}-u\right|^{2}\left(\frac{\alpha}{2}-L_{s}-\frac{C_{h}^{2}}{2}\right) d x d s\right]+\mathbb{E}\left[\int_{Q} \frac{\alpha}{2} e^{-\alpha s}|u|^{2} d x d s\right] \\
& +\mathbb{E}\left[\int_{Q} e^{-\alpha s}\left\{\frac{1}{2} h^{2}(u)-u \bar{w}_{s}-h(u) \bar{h}\right\} d x d s\right] .
\end{aligned}
$$

Now, by choosing $\alpha$ such that $\frac{\alpha}{2}-L_{s}-\frac{c_{h}^{2}}{2} \geqslant 0$, one gets that

$$
\liminf A_{\epsilon} \geqslant \mathbb{E}\left[\int_{Q} e^{-\alpha s}\left\{\frac{\alpha}{2}|u|^{2}-u \bar{w}_{s}+\frac{1}{2} h^{2}(u)-h(u) \bar{h}\right\} d x d s\right] .
$$

Using this in (3.3), we obtain

$$
\begin{aligned}
& \limsup \left(e^{-\alpha T} \frac{1}{2} \mathbb{E}\left[\left\|u_{\epsilon}(T)\right\|^{2}\right]+\mathbb{E}\left[\int_{Q} e^{-\alpha s}\left|\nabla u_{\epsilon}\right|^{2} d x d s\right]\right) \\
& +\mathbb{E}\left[\int_{Q} e^{-\alpha s}\left\{\xi_{2}+\frac{\alpha}{2}|u|^{2}-u \bar{w}_{s}+\frac{1}{2} h^{2}(u)-h(u) \bar{h}-f u\right\} d x d s\right] \\
& \leqslant \frac{1}{2}\left\|u_{0}\right\|^{2} .
\end{aligned}
$$

Besides, by applying the Itô formula to the process $u$ and the function $F(t, v)=\frac{1}{2} e^{-\alpha t}\|v\|^{2}$, we get, after taking the expectation,

$$
\begin{aligned}
& e^{-\alpha T} \frac{1}{2} \mathbb{E}\left[\|u(T)\|^{2}\right]-\frac{1}{2}\left\|u_{0}\right\|^{2}+\mathbb{E}\left[\int_{Q} e^{-\alpha s}|\nabla u|^{2} d x d s\right] \\
= & \mathbb{E}\left[\int_{Q} e^{-\alpha s}\left\{-\frac{\alpha}{2}|u|^{2}+u \bar{w}_{s}+\frac{1}{2} \bar{h}^{2}-\xi u+f u\right\} d x d s\right] .
\end{aligned}
$$

And by injecting it in (3.4), we get

$$
\begin{aligned}
& \lim \sup \left(e^{-\alpha T} \frac{1}{2} \mathbb{E}\left[\left\|u_{\epsilon}(T)\right\|^{2}\right]+\mathbb{E}\left[\int_{Q} e^{-\alpha s}\left|\nabla u_{\epsilon}\right|^{2} d x d s\right]\right) \\
& +\mathbb{E}\left[\int_{Q} e^{-\alpha s}\left\{\xi_{2}-\xi u+\frac{1}{2}(h(u)-\bar{h})^{2}\right\} d x d s\right] \\
& \leqslant e^{-\alpha T} \frac{1}{2} \mathbb{E}\left[\|u(T)\|^{2}\right]+\mathbb{E}\left[\int_{Q} e^{-\alpha s}|\nabla u|^{2} d x d s\right] .
\end{aligned}
$$


Thanks to the properties of the weak convergence and of the lim sup and lim inf, one has that

$$
\begin{aligned}
& e^{-\alpha T} \frac{1}{2} \mathbb{E}\left[\|u(T)\|^{2}\right]+\mathbb{E}\left[\int_{Q} e^{-\alpha s}|\nabla u|^{2} d x d s\right] \\
& \leq \liminf \left(e^{-\alpha T} \frac{1}{2} \mathbb{E}\left[\left\|u_{\epsilon}(T)\right\|^{2}\right]\right)+\liminf \left(\mathbb{E}\left[\int_{Q} e^{-\alpha s}\left|\nabla u_{\epsilon}\right|^{2} d x d s\right]\right) \\
& \leq \lim \sup \left(e^{-\alpha T} \frac{1}{2} \mathbb{E}\left[\left\|u_{\epsilon}(T)\right\|^{2}\right]+\mathbb{E}\left[\int_{Q} e^{-\alpha s}\left|\nabla u_{\epsilon}\right|^{2} d x d s\right]\right),
\end{aligned}
$$

so that

$$
\mathbb{E}\left[\int_{Q} e^{-\alpha s}\left\{\xi_{2}-\xi u+\frac{1}{2}(h(u)-\bar{h})^{2}\right\} d x d s\right] \leq 0
$$

Thanks to Proposition 3.9 and Remark 3.7, we note that for almost all $(\omega, x, t) \in \Omega \times Q, 0 \leqslant u(\omega, x, t) \leqslant 1$ and $\xi=\xi_{1}+\xi_{2}$ with $\xi_{1} \leq 0$ and $\xi_{2} \geq 0$.

Then $\xi_{2}-\xi u=(1-u) \xi_{2}-\xi_{1} u \geq 0$, and a first consequence of the previous inequality is that $h(u)=\bar{h}$. A second consequence is that $\xi_{2}-\xi u=(1-u) \xi_{2}-\xi_{1} u=0$ and because

- if $u=0$ then $\xi_{2}=0$. So $\xi=\xi_{1} \leqslant 0$ and in this way $\xi \in \mathbb{R}^{-}$,

- if $u=1$ then $\xi_{1}=0$. So $\xi=\xi_{2} \geqslant 0$ and in this way $\xi \in \mathbb{R}^{+}$, and

- if $0<u<1$, then $\xi_{1}=\xi_{2}=0$ and $\xi=0$,

one may conclude that $\xi \in \partial I_{[0,1]}(u)$.

The last consequence is that

$$
\begin{aligned}
& \lim \sup \left(e^{-\alpha T} \frac{1}{2} \mathbb{E}\left[\left\|u_{\epsilon}(T)\right\|^{2}\right]+\mathbb{E}\left[\int_{Q} e^{-\alpha s}\left|\nabla u_{\epsilon}\right|^{2} d x d s\right]\right) \\
& =e^{-\alpha T} \frac{1}{2} \mathbb{E}\left[\|u(T)\|^{2}\right]+\mathbb{E}\left[\int_{Q} e^{-\alpha s}|\nabla u|^{2} d x d s\right] .
\end{aligned}
$$

A standard monotone argument yields

$$
u_{\epsilon}(T) \rightarrow u(T) \text { in } L^{2}(\Omega \times D), \quad \nabla u_{\epsilon} \rightarrow \nabla u \text { in } L^{2}\left((0, T) \times \Omega, L^{2}(D)\right)
$$

Note that because $T$ is arbitrary, one can also conclude the convergence of $u_{\epsilon}(t)$ to $u(t)$ in $L^{2}(\Omega \times D)$ for any $t$. Then, by Proposition 2.2 and the lower semi-continuity of the norm for the weak-* convergence, $\mathbb{E}\left[\left\|u_{\epsilon}(t)\right\|^{2}\right]$ is bounded uniformly with respect to $t$ and $\epsilon$. Lebesgue converging theorem yields $u_{\epsilon} \rightarrow u$ in $L^{2}\left((0, T) \times \Omega, H^{1}(D)\right)$.

Then, by using the Lipschitz property of $w_{s}$, one gets that $\bar{w}_{s}=w_{s}(u)$ and that the convergence of $w_{s}\left(u_{\epsilon}\right)$ towards $w_{s}(u)$ holds strongly in $L^{2}(\Omega \times Q)$.

\subsection{Uniqueness of the solution}

Theorem 3.12

Problem (1.1) admits a unique solution.

Proof

We consider two solutions $u$ and $\hat{u}$ of Problem (1.1) associated with the same initial condition $u(0)=\hat{u}(0)=u_{0}$ :

$$
\begin{aligned}
d u+(\xi-\Delta u) d t & =\left(w_{s}(u)+f\right) d t+h(u) d W, \quad \xi \in \partial I_{[0,1]}(u), \\
d \hat{u}+(\hat{\xi}-\Delta \hat{u}) d t=\left(w_{s}(\hat{u})+f\right) d t+h(\hat{u}) d W, & \hat{\xi} \in \partial I_{[0,1]}(\hat{u}) .
\end{aligned}
$$

Note that, by monotonicity, it holds $(\xi-\hat{\xi})(u-\hat{u}) \geqslant 0$.

Besides, by applying the Itô formula to the process $u-\hat{u}$ and to the function $F(t, v)=e^{-\alpha s}\|v\|^{2}$ where $\alpha>0$, one gets after taking the expectation

$$
\begin{aligned}
& \frac{1}{2} e^{-\alpha T} \mathbb{E}\left[\left.||(u-\hat{u})(T)\right|^{2}\right]+\mathbb{E}\left[\int_{Q} e^{-\alpha s}|\nabla(u-\hat{u})|^{2} d x d s\right]+\frac{\alpha}{2} \mathbb{E}\left[\int_{Q} e^{-\alpha s}|u-\hat{u}|^{2} d x d s\right] \\
\leqslant & L_{s} \mathbb{E}\left[\int_{Q} e^{-\alpha s}|u-\hat{u}|^{2} d x d s\right]+\frac{C_{h}^{2}}{2} \mathbb{E}\left[\int_{Q} e^{-\alpha s}|u-\hat{u}|^{2} d x d s\right] .
\end{aligned}
$$

Then, by choosing $\alpha$ such that $\frac{\alpha}{2}-L_{s}-\frac{C_{h}^{2}}{2} \leqslant 0$, one gets that $u=\hat{u}$, and, going back to the equations, one has finally that $\xi=\hat{\xi}$ and the solution is unique. 


\section{References}

1. Brézis H. Opérateurs Maximaux Monotones et Semi-groupes de Contractions dans les Espaces de Hilbert, Number 5 in North Holland Math Studies: North-Holland Publishing Co., Amsterdam, 1973.

2. Da Prato G, Zabczyk J. Stochastic Equations in Infinite Dimensions, Encyclopedia of Mathematics and its Applications, vol. 44. Cambridge University Press: Cambridge, 1992.

3. Allen SM, Cahn JW. A microscopic theory for antiphase boundary motion and its application to antiphase domain coarsening. Acta Metallurgica 1979; 27(6):1085-1095.

4. Cherfils L, Miranville A, Zelik S. The Cahn-Hilliard equation with logarithmic potentials. Milan Journal of Mathematics 2011; 79(2):561-596.

5. Colli P, Gilardi G, Podio-Guidugli P, Sprekels J. Global existence and uniqueness for a singular/degenerate Cahn-Hilliard system with viscosity. Journal of Differential Equations 2013; 254(11):4217-4244.

6. Del Pino M, Kowalczyk M, Pacard F, Wei J. Multiple-end solutions to the Allen-Cahn equation in R2. Journal of Functional Analysis 2010; 258(2): 458-503.

7. Ward MJ. Metastable bubble solutions for the Allen-Cahn equation with mass conservation. SIAM Journal on Applied Mathematics 1996; 56(5): 1247-1279.

8. Antonopoulou DC, Karali G, Millet A. Existence and regularity of solution for stochastic Cahn-Hilliard/Allen-Cahn equation with unbounded noise diffusion. Journal of Differential Equations 2016; 260:2383-2417.

9. Ryser MD, Nigam N, Tupper PF. On the well-posedness of the stochastic Allen-Cahn equation in two dimensions. Journal of Computational Physics 2012; 231(6):2537-2550.

10. Barbu V, Rascanu A. Parabolic variational inequalities with singular inputs. Differential Integral Equations 1997; 10(1):67-83.

11. Bensoussan A, Rascanu A. Stochastic variational inequalities in infinite dimensional spaces. Numerical Functional Analysis and Optimization 1997; 18(1-2):19-54.

12. Rascanu A. Deterministic and stochastic differential equations in Hilbert spaces involving multivalued maximal monotone operators. PanAmerican Mathematical Journal 1996; 6(3):83-119.

13. Barbu V, Da Prato G. Irreducibility of the transition semigroup associated with the stochastic obstacle problem. Infinite Dimensional Analysis, Quantum Probability and Related Topics 2005; 8(3):397-406.

14. Frémond M. Non-smooth Thermomechanics. Springer-Verlag: Berlin, 2002.

15. Bonetti E, Bonfanti G, Rossi R. Global existence for a contact problem with adhesion. Mathematical Methods in the Applied Sciences 2008; 31: 1029-1064.

16. Bonetti E, Bonfanti G, Rossi R. Well-posedness and long-time behaviour for a model of contact with adhesion. Indiana University Mathematics Journal 2007; 56:2787-2819.

17. Bonetti E, Bonfanti G, Rossi R. Analysis of a unilateral contact problem taking into account adhesion and friction. Journal of Differential Equations 2012; 253:438-462.

18. Bonetti E, Schimperna G. Local existence for Frémond's model of damage in elastic materials. Continuum Mechanics and Thermodynamics 2004; 16:319-335.

19. Bonetti E, Schimperna G, Segatti A. On a doubly nonlinear model for the evolution of damaging in viscoelastic materials. Journal of Differential Equations 2005; 218:91-116.

20. Barbu V. Nonlinear Semigroups and Differential Equations in Banach Spaces. Noordhoff: Leyden, 1976.

21. Prévôt C, Röckner M. A Concise Course on Stochastic Partial Differential Equations, Lecture Notes in Mathematics, vol. 1905. Springer: Berlin, 2007. 